TRANSACTIONS OF THE

AMERICAN MATHEMATICAL SOCIETY

Volume 350, Number 6, June 1998, Pages 2487-2523

S $0002-9947(98) 01964-3$

\title{
ON THE DISTRIBUTION OF MASS IN COLLINEAR CENTRAL CONFIGURATIONS
}

\author{
PETER W. LINDSTROM
}

\begin{abstract}
Moulton's Theorem says that given an ordering of masses, $m_{1}, m_{2}$, $\ldots, m_{n}$, there exists a unique collinear central configuration with center of mass at the origin and moment of inertia equal to 1 . This theorem allows us to ask the questions: What is the distribution of mass in this standardized collinear central configuration? What is the behavior of the distribution as $n \rightarrow \infty$ ? In this paper, we define continuous configurations, prove a continuous version of Moulton's Theorem, and then, in the spirit of limit theorems in probability theory, prove that as $n \rightarrow \infty$, under rather general conditions, the discrete mass distributions of the standardized collinear central configurations have distribution functions which converge uniformly to the distribution function of the unique continuous standardized collinear central configuration which we determine.
\end{abstract}

\section{INTRODUCTION}

Central configurations are initial positions of masses that lead to special families of solutions of the $n$-body problem. They have been studied extensively, at least in part because the general $n$-body problem appears intractable for $n \geq 3$. (For a comprehensive introduction see Moeckel [5], and Saari [8].) The collinear central configurations were first studied by Euler [2] in the 1760s; and Moulton [7], at the turn of this century, proved an existence and uniqueness theorem for these configurations. Many recent papers have been published and new questions raised. Moeckel in the 1980s asked about the behavior of the mass distribution in the collinear case as $n \rightarrow \infty$. In 1990, attacking this problem, Buck [1] obtained bounds for the size of the configuration in the case of equal masses.

In this paper we present a solution to the mass distribution problem that was motivated by a probabilistic view. Taking the total mass of the point masses to be 1 , we can identify a configuration as a discrete probability distribution. Moulton's Theorem implies, if the $n$ masses are ordered from left to right on the $x$ axis, there is a unique positioning or configuration of the masses so that the associated probability distribution is standardized (i.e. the mean is 0 and the standard deviation is 1) and the configuration is central. In the work that follows, we will define collinear central configurations for continuous mass distributions, prove a continuous version of Moulton's Theorem, and then show that, if the ratio of the maximum mass to the minimum mass is kept less than some fixed value, as $n \rightarrow \infty$, the mass (or probability) distribution functions for the discrete standardized collinear central configurations converge uniformly to the continuous distribution function of the

Received by the editors October 1, 1995 and, in revised form, September 20, 1996.

1991 Mathematics Subject Classification. Primary 70F10.

(C)1998 American Mathematical Society 
standardized continuous collinear central configuration. We also show that the limiting distribution function corresponds to a quadratic density function which we determine.

In the case of equal masses, the convergence is quite rapid, with close agreement to the limit function for $n$ as small as 4 . This enables one to use the continuous collinear central configuration to estimate the potential and positions of the masses in the discrete case. Even for non-equal masses the predictions of position and potential are often quite good for small $n$. this is under further study in conjunction with other limiting distributions that arise when a few of the masses are kept fixed and not allowed to tend to 0 when $n$ gets large.

The uniform convergence result also shows promise of extensions to higher dimensions. See Lindstrom [3].

Since we are dealing only with collinear configurations, the notation that we now introduce will be limited to handling situations in $R^{1}$.

Definition 1.1. A (discrete) configuration $X=\left\{x_{1}, x_{2}, \ldots, x_{n} ; a_{1}, a_{2}, \ldots, a_{n}\right\}$ of $n$ bodies is a choice of positions $x_{1}, x_{2}, \ldots, x_{n} \in R^{1}$ and masses $a_{1}, a_{2}, \ldots, a_{n} \in R^{1}$. It will be assumed that $x_{1}<x_{2}<\cdots<x_{n}$. Also, unless it is specifically or by context indicated to the contrary, it will be assumed that $\sum_{i=1}^{n} a_{i}=1$. This configuration may also be denoted by $X_{A}$ when it is necessary to emphasize the mass vector $A=\left(a_{1}, a_{2}, \ldots, a_{n}\right)$. Similarly, if lower case $y$ 's represent positions and lower case $b$ 's the masses, then the configuration will be denoted by either $Y$ or $Y_{B}$.

The potential of the configuration $X=X_{A}$ is denoted by $U(X)$ and defined as

$$
U(X)=\sum_{i<j} \frac{a_{i} a_{j}}{x_{j}-x_{i}} .
$$

If $X=X_{A}$ is an $n$ point configuration, its mass distribution function, $F(x)$, is defined as:

$$
\begin{gathered}
0: x<x_{1} \\
F(x)=\sum_{i=1}^{k} a_{i}: x_{k} \leq x<x_{k+1} ; k=1, \ldots, n-1 . \\
1: x \geq x_{n}
\end{gathered}
$$

If $g(x)$ is a function defined at the mass positions of $X=X_{A}$, then $g(X)$ is the configuration defined as:

$$
\left\{g\left(x_{1}\right), g\left(x_{2}\right), \ldots, g\left(x_{n}\right) ; a_{1}, a_{2}, \ldots, a_{n}\right\} .
$$

In particular, if $c$ is a real number, then

$$
X+c=\left\{x_{1}+c, x_{2}+c, \ldots, x_{n}+c ; a_{1}, a_{2}, \ldots, a_{n}\right\}
$$

and

$$
c X=\left\{c x_{1}, c x_{2}, \ldots, c x_{n} ; a_{1}, a_{2}, \ldots, a_{n}\right\} .
$$

Let $X=X_{A}$. The mean or center of mass of the configuration $g(X)$, denoted by $E g(X)$ or $\mu(g(X))$, is defined as:

$$
E g(X)=\sum_{i=1}^{n} a_{i} g\left(x_{i}\right)
$$


The standard deviation of a configuration $X$, denoted $\sigma(X)$, is defined as:

$$
\sigma(X)=\sqrt{E X^{2}-(E X)^{2}} .
$$

A configuration $X=X_{A}$ is said to be standardized if $\sigma(X)=1$ and $\mu(X)=0$.

In the $n$-body problem of Newtonian mechanics, let $X_{A}$ represent the initial configuration of $n$ collinear bodies at rest, and $X(t)$ the configuration at a future time $t . X_{A}$ is a collinear central configuration if there is some decreasing function $\varphi(t)$ and time $\tau>0$ such that $\varphi(0)=1, \varphi(\tau)=0$, and, for $0 \leq t \leq \tau, X(t)=$ $\mu\left(X_{A}\right)+\varphi(t)\left(X_{A}-\mu\left(X_{A}\right)\right)$. That is, $X_{A}$ is a collinear central configuration, if starting at rest it collapses homothetically to its center of mass and results in a collision singularity. Collinear central configurations which are standardized will be denoted by a superscript asterisk.

We will not be involved with the $n$-body problem per se, but rather will focus on collinear central configurations for their own sake. Our analysis will be based upon a reworded version of Moulton's Theorem which includes an alternative characterization of collinear central configurations in terms of the potential function $U(X)$.

Theorem 1.1 (Moulton's Theorem). Given an $n$-dimensional mass vector $A$, there exists a unique standardized collinear central configuration $X_{A}^{*}$. The positions of the bodies in $X_{A}^{*}$ are those that minimize $U\left(X_{A}\right)$ subject to the constraints $\mu\left(X_{A}\right)=0$ and $\sigma\left(X_{A}\right)=1$. (See the proof in [7] and further discussions and proofs in [4], [5], and [8].)

For completeness sake, we note that the property of being a collinear central configuration is maintained by translation and change of scale, and hence from Moulton's Theorem, it follows that all collinear central configurations corresponding to a mass vector $A$ are of the form $c X_{A}^{*}+b$ where $b$ and $c$ are scalars and $c>0$.

From this point on our efforts will be aimed at analyzing $X_{A}^{*}$ and its mass distribution when $n$ is large.

\section{Continuous analogues}

As in the case of probability distributions, when $n$ is large we can use a histogram to picture the mass distribution in a configuration $X$. The motivating idea behind this paper was the assumption that as $n$ gets large, at least in the case of equal masses, the histograms for $X_{A}^{*}$ would approach some function $f^{*}(x)$. Further it seemed likely that $f^{*}(x)$ would correspond to a continuous mass distribution with some sort of minimal potential.

An obvious candidate for the potential of a distribution with density $f(x)$ is

$$
\int_{-\infty}^{\infty} \int_{y}^{\infty} \frac{f(x) f(y) d x d y}{x-y}
$$

The problem with this integral is that it is generally divergent. However, a useful modification is possible.

Looking to the discrete potential,

$$
U(X)=\sum_{i<j} \frac{a_{i} a_{j}}{x_{j}-x_{i}}
$$

for guidance, we note that with an $n$-point configuration restricted to a bounded interval, the condition $j>i$ would, for low potential configurations, probably imply that $x_{j}>x_{i}+\frac{c}{n}$ for some fixed $c$. 
Now, if $f$ were defined by a histogram and we wanted to approximate the discrete potential, then, since a point mass is smeared over a small interval by the histogram, a reasonable analogue to $x_{j}>x_{i}+\frac{c}{n}$ might be $x>y+\frac{c}{n}$. This suggests that we examine

$$
\int_{-\infty}^{\infty} \int_{y+\frac{c}{n}}^{\infty} \frac{f(x) f(y) d x d y}{x-y}
$$

Under general conditions on $f$, its behavior as $n \rightarrow \infty$ is determined by the next proposition.

Proposition 2.1. Let $f(x)$ be non-negative on $(-\infty, \infty)$ and have a set of discontinuities which has measure 0 . Then, if

$$
\int_{-\infty}^{\infty} f^{2}(x) d x<\infty, \int_{-\infty}^{\infty} f(x) d x<\infty
$$

and $c>0$ is fixed,

$$
\operatorname{Lim}_{n \rightarrow \infty} \frac{\int_{-\infty}^{\infty} \int_{y+\frac{c}{n}}^{\infty} \frac{f(x) f(y) d x d y}{x-y}}{\log (n)}=\int_{-\infty}^{\infty} f^{2}(x) d x .
$$

Proof. (See Appendix A.)

Proposition 2.1 suggests that we use $\int_{-\infty}^{\infty} f^{2}(x) d x$ to measure the potential of a continuous mass distribution. It further suggests that as $n \rightarrow \infty$, if the histograms associated with $X_{A}$ were to approach $f$, that $\frac{U\left(X_{A}\right)}{\log (n)}$ would remain bounded and approach $\int_{-\infty}^{\infty} f^{2}(x) d x$. These considerations together with the hypotheses of Proposition 2.1 motivate the following definition statement. $L_{2}(R)$ will denote the Hilbert space of square integrable functions on $(-\infty, \infty)$, and $\|f\|_{2}=\sqrt{\int_{-\infty}^{\infty} f^{2}(x) d x}$ the $L_{2}$ norm of $f \in L_{2}(R)$.

Definition 2.1. A (mass) density function is defined as a function $f \in L_{2}(R)$ with the following properties:

(i) $f$ is non-negative a.e.,

(ii) $\int_{-\infty}^{\infty} f(x) d x=1$.

The set of standardized density functions, denoted $D(R)$, is defined as those density functions with the following properties:

(iii) $\int_{-\infty}^{\infty} x f(x) d x=0$,

(iv) $\int_{-\infty}^{\infty} x^{2} f(x) d x=1$.

A continuous configuration $X$ is defined as a distribution of mass on $(-\infty, \infty)$ with the property that there is a density function $f$ such that the mass $X$ in any interval $I$ is given by $\int_{I} f(x) d x$.

If $X$ is a continuous configuration with density function $f$, the normalized potential of $X$, denoted $\Pi(X)$, is defined as: $\Pi(X)=\|f\|_{2}^{2}$.

If $X$ is an $n$-point configuration, the normalized potential of $X$, denoted $\Pi(X)$, is defined as:

$$
\Pi(X)=\frac{U(X)}{\log (n)} .
$$


If $X$ is a continuous configuration with density function $f$, and $g$ is a real valued function defined on $(-\infty, \infty)$, then $E g(X)$ is defined as

$$
E g(X)=\int_{-\infty}^{\infty} g(x) f(x) d x
$$

The center of mass of a continuous configuration $X$, denoted $\mu(X)$, is defined as:

$$
\mu(X)=E X .
$$

The standard deviation of a continuous configuration $X$ is denoted and defined in terms of $E$ as in Definition 1.1.

A continuous configuration $X$ is standardized if its density function is contained in $D(R)$, or equivalently, $\mu(X)=0$ and $\sigma(X)=1$. The set of standardized continuous configurations is denoted $S_{\infty}$.

A continuous configuration $X$, having a mean and standard deviation, is a continuous collinear central configuration if

$$
\Pi(X)=\underset{Y: \sigma(Y)=\sigma(X)}{\operatorname{Min}}[\Pi(Y)],
$$

where $Y$ denotes a continuous configuration. Continuous collinear central configurations which are standardized will be denoted by a superscript asterisk.

If $X$ is a continuous configuration with distribution function $F(x)$ and density function $f(x)$, then for any real $c, X+c$ is the continuous configuration with distribution function $F(x-c)$ and density function $f(x-c)$. Also, if $c>0, c X$ is the continuous configuration with distribution function $F(x / c)$ and density function $f(x / c) / c$.

If $X$ is any configuration, discrete or continuous, with mean $\mu$ and standard deviation $\sigma$, the standardization of $X$, denoted $X^{s}$, is defined as:

$$
X^{s}=\frac{X-\mu}{\sigma} \text {. }
$$

Two useful consequences of the above definitions are:

(i) We can manipulate discrete or continuous configurations algebraically in the sense that if $c>0$ and $b$ are real numbers and $Y=c X+b$, then $X=(Y-b) / c$.

(ii) If $c>0$, then $\sigma(c X+b)=c \sigma(X)$.

Using the normalized potential rather than $U(X)$ enables us, in many instances, to carry results across from discrete to continuous configurations.

Proposition 2.2. If $X$ is any configuration, discrete or continuous, and $c>0$ and $b$ are real numbers, then

$$
\Pi(c X+b)=\frac{\Pi(X)}{c} .
$$

Proof. The result is obvious if $X$ is discrete. If $X$ is continuous with density function $f(x)$, then the density function for $c X+b$ is equal to $\frac{f\left(\frac{x-b}{c}\right)}{c}$. Hence,

$$
\Pi(c X+b)=\frac{1}{c^{2}} \int_{-\infty}^{\infty} f^{2}\left(\frac{x-b}{c}\right) d x=\frac{1}{c} \int_{-\infty}^{\infty} f^{2}(u) d u=\frac{\Pi(X)}{c} .
$$

Proposition 2.3. If $X$ is any configuration, discrete or continuous, with mean $\mu$ and standard deviation $\sigma$, then

$$
\Pi\left(X^{s}\right)=\sigma \Pi(X) .
$$


Proof. The result is an immediate consequence of Proposition 2.2.

Proposition 2.3 is used in the proof of the following continuous version of Moulton's Theorem.

Theorem 2.1. There exists a unique standardized continuous collinear central configuration $X^{*}$. The density function, $f^{*}(x)$, for $X^{*}$ is given by

$$
f^{*}(x)=\left\{\begin{array}{l}
\frac{3 \sqrt{5}\left(1-\frac{x^{2}}{5}\right)}{20}:|x| \leq \sqrt{5}, \\
0:|x|>\sqrt{5}
\end{array}\right.
$$

Proof. (See sections 3-5.)

As in the discrete case, the standardized collinear central configuration determines all other continuous collinear central configurations. It is easy to show that a continuous configuration is a collinear central configuration if and only if it is of the form $c X^{*}+b$ where $c>0$ and $b$ are real numbers.

\section{Proof of Moulton's Theorem for continuous configurations, Part I}

We begin with a definition statement.

Definition 3.1 (Notation). The infimum of $\Pi(X)$, over all $X$ contained in $S_{\infty}$, is denoted as $\Pi^{*}$.

If $a>0$, the set of density functions in $D(R)$ which vanish a.e. outside of $[-a, a]$ is denoted as $D([-a, a])$.

The set of configurations in $S_{\infty}$ which vanish outside of $[-a, a]$, denoted $S_{\infty}([a, a])$, is defined as those configurations with density functions in $D([-a, a])$.

The infimum of $\Pi(X)$, over all $X$ contained in $S_{\infty}([-a, a])$, is denoted as $\Pi_{a}^{*}$.

We first establish that $\Pi^{*}$ is positive. The $\sqrt{5}$ appearing in the proof arises subsequently in a natural way.

Proposition 3.1. $\Pi^{*} \geq \frac{32}{\sqrt{5}}$.

Proof. Let $X$ be contained in $S_{\infty}$ and have density function $f$.

Setting $r=\int_{|x|>\sqrt{5}} f(x) d x$, since $1=\sigma^{2}(X)=\int_{-\infty}^{\infty} x^{2} f(x) d x$, we have

$$
1 \geq \int_{|x|>\sqrt{5}} x^{2} f(x) d x \geq 5 \int_{|x|>\sqrt{5}} f(x) d x=5 r .
$$

Thus, $r \leq .2$.

From the Schwarz inequality,

$$
(1-r)^{2}=\left(\int_{-\sqrt{5}}^{\sqrt{5}} f(x) d x\right)^{2} \leq\left(\int_{-\sqrt{5}}^{\sqrt{5}} f^{2}(x) d x\right)\left(\int_{-\sqrt{5}}^{\sqrt{5}} 1 d x\right) \leq 2 \sqrt{5} \Pi(X) .
$$

Hence, $\Pi(X) \geq \frac{(.8)^{2}}{2 \sqrt{5}}$.

In proving Theorem 2.1, we must show that there exists a unique element in $S_{\infty}$ with normalized potential equal to $\Pi^{*}$. As a beginning, we solve the analogous problem for $S_{\infty}([-a, a])$.

Proposition 3.2. If $S_{\infty}([-a, a])$ is non-empty, there is a unique configuration in $S_{\infty}([-a, a])$ with normalized potential equal to $\Pi_{a}^{*}$. 
Proof. To establish the result, we need to show in $D([-a, a])$ there exists a unique element of minimum $L_{2}$ norm. To do this we will make use of the fact that in a complete inner product space there is a unique element of minimum norm in any non-empty, closed, convex subset. The proof will be complete if we show that a non-empty $D([-a, a])$ is a closed convex subset of $L_{2}(R)$.

Convexity. If $f, g$ vanish a.e. outside of $[-a, a]$ and satisfy (i)-(iv) in Definition 2.1, then, for $0 \leq \lambda \leq 1, \lambda f+(1-\lambda) g$ also has these same properties.

Closure. Let $L_{2}([-a, a])$ denote the subspace of $L_{2}(R)$ consisting of those functions vanishing a.e. outside of $[-a, a] . L_{2}([-a, a])$ is itself a Hilbert space.

If $f_{n} \in D([-a, a])$ and $\left\|f_{n}-f\right\|_{2} \rightarrow 0$, since $\int_{-\infty}^{\infty} x^{2} f(x) d x, \int_{-\infty}^{\infty} x f(x) d x$, and $\int_{-\infty}^{\infty} f(x) d x$ is each a continuous linear functional defined for $f$ contained in $L_{2}([-a, a])$, it follows by the completeness of $L_{2}([-a, a])$ and the continuity of the functionals that $f$ is contained in $L_{2}([-a, a])$ and satisfies properties (ii)-(iv) in Definition 2.1. Noting that the set of functions in $L_{2}([-a, a])$ which are non-negative a.e. is a closed subset, we conclude that $f$ is non-negative a.e. and hence is contained in $D([-a, a])$.

Definition 3.2 (Notation). The configuration in $S_{\infty}([-a, a])$ with normalized potential equal to $\Pi_{a}^{*}$ is denoted by $X_{a}^{*}$. The density function corresponding to $X_{a}^{*}$ is denoted by $f_{a}^{*}$.

The truncated 1 and $x^{2}$ functions are denoted $g_{0 a}$ and $g_{2 a}$ and defined as:

$$
\begin{aligned}
& g_{0 a}(x)=\begin{array}{l}
1:|x| \leq a, \\
0:|x|>a,
\end{array} \\
& g_{2 a}(x)=\begin{array}{c}
x^{2}:|x| \leq a, \\
0:|x|>a .
\end{array}
\end{aligned}
$$

The subspace of $L_{2}([-a, a])$, of truncated even quadratics, spanned by $g_{0 a}$ and $g_{2 a}$ is denoted by $Q([-a, a])$.

If $D(R)$ were closed, we could extend Proposition 3.2 to $S_{\infty}$ thereby providing the existence and uniqueness portion of the continuous version of Moulton's Theorem. However, we have not established closure and believe it does not hold. Our approach is to try and determine $f_{a}^{*}$, so as to possibly show $\Pi_{a}^{*}=\Pi^{*}$ for some $a$.

Proposition 3.3. If $Q([-a, a]) \cap D([-a, a])$ is non-empty, the intersection consists of the single element $f_{a}^{*}$.

Proof. Let $g=c_{0} g_{0 a}+c_{2} g_{2 a}$ be contained in $Q([-a, a]) \cap D([-a, a])$ and let $f$ be any other element in $D([-a, a])$. With $\langle q, p\rangle$ denoting the inner product $\int_{-\infty}^{\infty} q(x) p(x) d x$, we note that $\left\langle g_{i a}, f-g\right\rangle=0, i=0,2$, and hence, $\langle g, f-g\rangle=0$. Since $g$ and $f-g$ are orthogonal, the Pythagorean Theorem yields $\|f\|_{2}^{2}=\|(f-g)+g\|_{2}^{2}=\|f-g\|_{2}^{2}+\|g\|_{2}^{2}$. Hence, $\|f\|_{2}^{2}>\|g\|_{2}^{2}$, unless $f=g$. This implies that there is a unique element in $Q([-a, a]) \cap D([-a, a])$, and it has the smallest norm of any element in $D([-a, a])$. Thus the intersection consists solely of $f_{a}^{*}$.

Some analysis reveals that the largest value of $a$ for which $Q([-a, a]) \cap D([-a, a])$ is non-empty is $a=\sqrt{5}$. Though we make no essential use of this fact, it does suggest that $\sqrt{5}$ plays a special role in the problem. 
Proposition 3.4. $\Pi_{\sqrt{5}}^{*}=\frac{.6}{\sqrt{5}}$ and $f_{\sqrt{5}}^{*}$ is given by,

$$
f_{\sqrt{5}}^{*}(x)=\left\{\begin{array}{l}
\frac{3 \sqrt{5}\left(1-\frac{x^{2}}{5}\right)}{20}:|x| \leq \sqrt{5}, \\
0:|x|>\sqrt{5} .
\end{array}\right.
$$

Proof. In order for $Q([-a, a]) \cap D([-a, a])$ to be non-empty there must exist $c_{0}, c_{1}$ such that:

(i) $c_{0} g_{0 a}+c_{2} g_{2 a}$ is non-negative on $[-a, a]$,

(ii) $\int_{-a}^{a}\left(c_{0} g_{0 a}(x)+c_{2} g_{2 a}(x)\right) d x=1$,

(iii) $\int_{-a}^{a}\left(c_{0} g_{0 a}(x)+c_{2} g_{2 a}(x)\right) x d x=0$,

(iv) $\int_{-a}^{a}\left(c_{0} g_{0 a}(x)+c_{2} g_{2 a}(x)\right) x^{2} d x=1$.

Since (iii) is always satisfied, taking $a=\sqrt{5}$ and solving (ii) and (iv) yields $c_{0}=\frac{3 \sqrt{5}}{20}$ and $c_{2}=\frac{-3 \sqrt{5}}{100}$. The resulting function is non-negative and hence, $f_{\sqrt{5}}^{*}=c_{0} g_{0 a}+$ $c_{2} g_{2 a}$. Computation then gives $\Pi_{\sqrt{5}}^{*}=\int_{-\sqrt{5}}^{\sqrt{5}}\left(c_{0} g_{0 \sqrt{5}}(x)+c_{2} g_{2 \sqrt{5}}(x)\right)^{2} d x=\frac{.6}{\sqrt{5}}$.

Before completing the proof of the continuous version of Moulton's Theorem we will examine a transformation that will be used in the proof.

\section{The truncation transformation For CONTINUOUS CONFIGURATIONS}

This transformation is used to show that if a standardized continuous configuration extends beyond $[-\sqrt{5}, \sqrt{5}]$, it is possible to find another standardized continuous configuration with a lower normalized potential.

Definition 4.1. Let $X \in S_{\infty}$ exist with density function $f$; then $r_{i}(X), i=0,1,2$, are defined as:

$$
r_{i}(X)=\int_{|x|>\sqrt{5}} x^{i} f(x) d x .
$$

Proposition 4.1. For each standardized configuration, continuous or discrete, the mass outside of the interval $[-\sqrt{5}, \sqrt{5}]$ is less than or equal to .2 .

Proof. Let $r$ denote the mass of $X$ outside of $[-\sqrt{5}, \sqrt{5}]$. The result follows from $1=\sigma^{2}(X)=E X^{2} \geq(\sqrt{5})^{2} r$.

Noting that Proposition 4.1 implies $1-r_{0}(X) \geq .8>0$, we define the truncation transformation as follows.

Definition 4.2. Let $X \in S_{\infty}$ exist with density function $f$. The truncation of $X$, denoted $T(X)$, is defined as the continuous configuration with density function $f_{T}$ given by:

$$
f_{T}(x)=\left\{\begin{array}{l}
\frac{f(x)}{1-r_{0}(x)}:|x| \leq \sqrt{5}, \\
0:|x|>\sqrt{5} .
\end{array}\right.
$$

The related function $W$ is defined by:

$$
W(r)=\frac{1-5 r}{(1-r)^{5}} .
$$


$T$ eliminates the mass of $X$ outside of $[-\sqrt{5}, \sqrt{5}]$ and rescales the remaining mass so that the total is again 1.

If $X \in S_{\infty}, T^{S}(X)$ represents the standardization of $T(X)$. Hence $T^{s}$ maps $S_{\infty}$ into $S_{\infty}$. The fixed points of $T^{S}$ are those configurations with no mass outside $[-\sqrt{5}, \sqrt{5}]$. The next proposition, which makes use of the function $W$, will show that $T^{s}$ maps configurations extending beyond $[-\sqrt{5}, \sqrt{5}]$ into configurations of lower normalized potential. This special property of reducing the normalized potential for configurations extending beyond the truncation interval would not hold if $T$ were defined to eliminate the mass outside of $[-a, a]$ where $a<\sqrt{5}$.

Lemma 4.1. $\sqrt{W(r)}$ is strictly decreasing on $[0, .2]$ and attains a maximum of 1 at $r=0$.

Proof. The result follows from the fact that $W^{\prime}(r)=\frac{-20 r}{(1-r)^{6}}$.

Proposition 4.2. For $X \in S_{\infty}, \Pi\left(T^{S}(X)\right) \leq \sqrt{W\left(r_{0}(X)\right)} \Pi(X)$.

Proof. Let $X \in S_{\infty}$ have density function $f$, and set $r_{i}=r_{i}(X)$. We have $\Pi(T(X))=\left\|f_{T}\right\|_{2}^{2}=\frac{1}{\left(1-r_{0}\right)^{2}} \int_{-\sqrt{5}}^{\sqrt{5}} f^{2}(x) d x \leq \frac{\|f\|_{2}^{2}}{\left(1-r_{0}\right)^{2}}=\frac{\Pi(X)}{\left(1-r_{0}\right)^{2}}$. Also,

$$
\begin{aligned}
\sigma^{2}(T(X)) & =\frac{1}{\left(1-r_{0}\right)} \int_{-\sqrt{5}}^{\sqrt{5}} x^{2} f(x) d x-\frac{1}{\left(1-r_{0}\right)^{2}}\left(\int_{-\sqrt{5}}^{\sqrt{5}} x f(x) d x\right)^{2} \\
& =\frac{1-r_{2}}{1-r_{0}}-\frac{\left(0-r_{1}\right)^{2}}{\left(1-r_{0}\right)^{2}}
\end{aligned}
$$

and thus, $\sigma(T(X)) \leq \sqrt{\frac{1-r_{2}}{1-r_{0}}}$.

Noting $r_{2}=\int_{|x|>\sqrt{5}} x^{2} f(x) d x \geq 5 r_{0}$ and from Proposition 2.3 that $\Pi\left(T^{S}(X)\right)=$ $\sigma(T(X)) \Pi(T(X))$, we conclude that

$$
\Pi\left(T^{S}(X)\right) \leq \sqrt{\frac{1-5 r_{0}}{1-r_{0}}} \Pi(T(X)) \leq \sqrt{W\left(r_{0}\right)} \Pi(X) .
$$

The next proposition will enable us to bound the location of $T^{S}(X)$ when $X \in$ $S_{\infty}$ has nearly minimal normalized potential.

Note that if $\left\{\alpha_{i}\right\}$ is a sequence, $\operatorname{Lim} \alpha_{i}=\operatorname{Lim}_{i \rightarrow \infty} \alpha_{i}$.

Lemma 4.2. If $\left\{X_{i}\right\}$ is a sequence of configurations contained in $S_{\infty}$ for which $\operatorname{Lim} \Pi\left(X_{i}\right)=\Pi^{*}$, then $\operatorname{Lim} r_{0}\left(X_{i}\right)=0$.

Proof. Set $r_{0 i}=r_{0}\left(X_{i}\right)$, and $Z_{i}=T^{S}\left(X_{i}\right)$. Proposition 4.2 yields $\Pi\left(Z_{i}\right) \leq$ $\sqrt{W\left(r_{0 i}\right)} \Pi\left(X_{i}\right)$. It follows from Lemma 4.1 that if $\operatorname{Lim} r_{0 i} \neq 0$, there will exist $j$ such that $\Pi\left(Z_{j}\right)<\Pi^{*}$. But this is impossible because $Z_{j} \in S_{\infty}$. Hence, $\operatorname{Lim} r_{0}\left(X_{i}\right)=0$.

Proposition 4.3. If $\left\{X_{i}\right\}$ is a sequence of configurations contained in $S_{\infty}$ for which $\operatorname{Lim} \Pi\left(X_{i}\right)=\Pi^{*}$, then $\operatorname{Lim} \sigma\left(T\left(X_{i}\right)\right)=1$ and $\operatorname{Lim} \mu\left(T\left(X_{i}\right)\right)=0$.

Proof. Let $Z_{i}=T^{S}\left(X_{i}\right)$. With $r_{j i}=r_{j}\left(X_{i}\right), j=0,1,2$, from the proof of Proposition 4.2 , we have

$$
\sigma^{2}\left(T\left(X_{i}\right)\right)=\frac{1-r_{2 i}}{1-r_{0 i}}-\frac{\left(0-r_{1 i}\right)^{2}}{\left(1-r_{0 i}\right)^{2}}
$$


and

$$
\Pi\left(Z_{i}\right) \leq \sqrt{\frac{1-r_{2 i}}{1-r_{0 i}}-\frac{\left(0-r_{1 i}\right)^{2}}{\left(1-r_{0 i}\right)^{2}}} \frac{\Pi\left(X_{i}\right)}{\left(1-r_{0 i}\right)^{2}} .
$$

Since Proposition 4.2 states that $\operatorname{Lim} r_{0 i}=0$, it follows that if either $\operatorname{Lim} r_{2 i} \neq 0$ or $\operatorname{Lim} r_{1 i} \neq 0$, then there will exist a $j$ such that $\Pi\left(Z_{j}\right)<\Pi^{*}$. But this is impossible. Hence, $\operatorname{Lim} r_{2 i}=0$ and $\operatorname{Lim} r_{1 i}=0$. Now using $\mu\left(T\left(X_{i}\right)\right)=\frac{-r_{1 i}}{1-r_{0 i}}$ and $\sigma\left(T\left(X_{i}\right)\right)=$ $\sqrt{\frac{1-r_{2 i}}{1-r_{0 i}}-\frac{\left(0-r_{1 i}\right)^{2}}{\left(1-r_{0 i}\right)^{2}}}$, we conclude that $\operatorname{Lim} \mu\left(T\left(X_{i}\right)\right)=0$ and $\operatorname{Lim} \sigma\left(T\left(X_{i}\right)\right)=1$.

\section{Proof of Moulton's Theorem for CONTINUOUS CONFIGURATIONS, PART II}

Using the truncation transformation, we can readily complete the proof of Theorem 2.1.

The following two propositions constitute the proof.

Proposition 5.1. If there exists a configuration $X^{*} \in S_{\infty}$ such that $\Pi\left(X^{*}\right)=\Pi^{*}$, then $X^{*}$ is unique and equals $X_{\sqrt{5}}^{*}$.

Proof. If $r_{0}\left(X^{*}\right)>0$, then Proposition 4.2 yields the contradiction $\Pi\left(T^{S}\left(X^{*}\right)\right)<$ $\Pi^{*}$. Thus $X^{*}$ is contained in $[-\sqrt{5}, \sqrt{5}]$. As a result, $\Pi^{*}=\Pi_{\sqrt{5}}^{*}$, and $X^{*}=X_{\sqrt{5}}^{*}$.

Proposition 5.2. There exists a configuration $X^{*} \in S_{\infty}$ such that $\Pi\left(X^{*}\right)=\Pi$.

Proof. Let $\left\{X_{i}\right\}$ be a sequence of configurations in $S_{\infty}$ for which $\operatorname{Lim} \Pi\left(X_{i}\right)=\Pi^{*}$. Since $T^{S}\left(X_{i}\right) \in S_{\infty}$, Proposition 4.2 implies that $\operatorname{Lim} \Pi\left(T^{S}\left(X_{i}\right)\right)=\Pi^{*}$. Further, since $T^{S}\left(X_{i}\right)$ is contained in the interval $|x| \leq \frac{\sqrt{5}+\mu\left(T\left(X_{i}\right)\right)}{\sigma\left(T\left(X_{i}\right)\right)}$, and since $\sigma\left(T\left(X_{i}\right)\right) \neq 0$, Proposition 4.3 implies that there exists $b>0$, such that for all $i, T^{S}\left(X_{i}\right)$ is contained in $[-b, b]$. It follows that $\Pi^{*}=\Pi_{b}^{*}$, and hence, $\Pi\left(X_{b}^{*}\right)=\Pi^{*}$.

\section{Uniform CONVERgence: The strategy}

The task is now to prove that under rather general conditions the mass distribution functions for discrete standardized collinear central configurations converge uniformly to $F^{*}$, the mass distribution function for $X^{*}$. In order to discuss the strategy for doing so, we require additional definitions and notation.

Definition 6.1. If $A$ is a mass vector, the dispersion of $A$, denoted by $d(A)$, is defined as:

$$
d(A)=\frac{\max _{i} a_{i}}{\min _{i} a_{i}}
$$

The dispersion of a configuration $X=X_{A}$ is defined to be $d(A)$.

The set of n-point mass-vectors having dispersion less than or equal to $K$ is denoted as $M_{n}[K]$.

The least upper bound of normalized potentials for n-point standardized collinear central configurations of dispersion less than or equal to $K$, denoted $\bar{\Pi}^{*}[K ; n]$, is defined as $\bar{\Pi}^{*}[K ; n]=\operatorname{Sup}\left[\Pi\left(X_{A}^{*}\right): A \in M_{n}[K]\right]$.

The greatest lower bound of normalized potentials for n-point standardized collinear central configurations of dispersion less than or equal to $K$, denoted $\underline{\Pi}^{*}[K ; n]$, is defined as $\underline{\Pi}^{*}[K ; n]=\operatorname{Inf}\left[\Pi\left(X_{A}^{*}\right): A \in M_{n}[K]\right]$. 
The upper asymptotic normalized potential for standardized collinear central configurations of dispersion less than or equal to $K$, denoted $\bar{\Pi}^{*}[K]$, is defined as $\bar{\Pi}^{*}[K]=\operatorname{LimSup}_{n \rightarrow \infty} \bar{\Pi}^{*}[K ; n]$.

The lower asymptotic normalized potential for standardized collinear central configurations of dispersion less than or equal to $K$, denoted $\underline{\Pi}^{*}[K]$, is defined as $\underline{\Pi}^{*}[K]=\operatorname{LimInf}_{n \rightarrow \infty} \underline{\Pi}^{*}[K ; n]$.

The uniform norm of $G(x)$, defined as $\operatorname{Sup}_{-\infty<x<\infty}|G(x)|$, is denoted by $\|G\|_{S}$.

Our goal is to establish uniform convergence in the case of configurations of bounded dispersion. The notion of dispersion has been introduced because simply assuming that the maximum mass in the configurations tends to 0 is not sufficient to establish the convergence. The key to our proof resides in showing:

(i) For fixed $K \geq 1, \bar{\Pi}^{*}[K]=\underline{\Pi}^{*}[K]=\Pi^{*}$, and hence, when the number of points in a standardized collinear central configuration of dispersion less than or equal to $K$ is large, its normalized potential is close to $\Pi^{*}$.

(ii) If $X \in S_{\infty}$ and $\Pi(X)$ is close to $\Pi^{*}$, then the mass distribution function for $X$ is uniformly close to $F^{*}$.

Let $n$ be large, $A_{n} \in M_{n}[K]$, and $X_{n}^{*}$ represent the standardized collinear central configuration corresponding to $A_{n}$. Then (i) and (ii) suggest the following approach for showing that the mass distribution function for $X_{n}^{*}$ is close to $F^{*}$.

Proof Strategy. Use (i) to conclude that $\Pi\left(X_{n}^{*}\right) \approx \Pi^{*}$, and then construct a standardized histogram-like configuration, $H_{n}^{S} \in S_{\infty}$, which approximates $X_{n}^{*}$ and for which $\Pi\left(H_{n}^{S}\right) \approx \Pi\left(X_{n}^{*}\right)$. Next, since $\Pi\left(H_{n}^{S}\right) \approx \Pi^{*}$, use (ii) to conclude that the mass distribution function for $H_{n}^{S}$ is uniformly close to $F^{*}$. Finally, on verifying that $H_{n}^{S}$ and $X_{n}^{*}$ have approximately the same mass distribution, conclude that the mass distribution function for $X_{n}^{*}$ is uniformly close to $F^{*}$.

In implementing this strategy there are technical difficulties that arise. In particular, if we attempt to construct the unstandardized histogram $H_{n}$ directly from $X_{n}^{*}$, we encounter difficulties due to the following:

1. If a standardized discrete configuration $X$ has long tails, an appropriate histogram constructed from $X$ could smear outlying point masses of $X$ over large intervals, thus altering the mean and standard deviation. As a result the standardized version of the histogram might not have a mass distribution close to that of the histogram, and hence, not close to that of $X$.

2. In the case when $X$ is an equal-mass configuration known to be contained in a given finite interval, we are able to use a method, based upon an inequality (Lemma 8.4) holding in the equal mass case, to show that the normalized potential of $X$ is close to that of the constructed histogram. The same approach fails if the masses of $X$ are not equal.

To surmount these difficulties, and others caused by lack of guarantee of uniform boundedness, and by non-equal masses, we alter $X_{n}^{*}$ slightly prior to constructing $H_{n}$. The alternation turns out to be non-existent in the sense of standardization and slight in the sense of mass distribution and normalized potential. Asymptotically in these senses, as $n \rightarrow \infty$, there is no alteration. We use configuration transformations to perform the alteration and do so as follows:

1. To guarantee uniform boundedness of the configurations prior to constructing the histograms, we use a discrete version of the truncation transformation $T$. We pass from $X_{n}^{*}$ to $Z_{n}=T^{S}\left(X_{n}^{*}\right)$. 
2. To enable us to use the equal-mass inequality, we coalesce neighboring point masses in $Z_{n}$ to form a new equal-mass configuration of fewer points. This configuration is denoted by $K_{T}\left(Z_{n}\right)$. We then pass from $Z_{n}$ to $W_{n}=K_{T}^{S}\left(Z_{n}\right)$.

The unstandardized histogram $H_{n}$ is constructed from equal-mass configuration $W_{n}$ and denoted $H\left(W_{n}\right)$. The standardized version, $H_{n}^{S}$, is defined as $H_{n}^{S}=$ $H^{S}\left(W_{n}\right)$. Procedure 6.1 summarizes the transformation process leading from the many-mass standardized colinear central configuration $X_{n}^{*}$ to the standardized histogram configuration $H_{n}^{S}$, which in mass distribution is close to both $X_{n}^{*}$ and $X^{*}$.

Procedure 6.1. $\left(X_{n}^{*}\right) \rightarrow\left(Z_{n}=T^{S}\left(X_{n}^{*}\right)\right) \rightarrow\left(W_{n}=K_{T}^{S}\left(Z_{n}\right)\right) \rightarrow\left(H_{n}^{S}\right)$.

In Sections 7-10, we give the specific definitions of these, and one other transformation, and derive the necessary properties. In Section 11, we prove (i) and (ii) and go on to implement the proof strategy outlined above.

\section{THE COALESCING TRANSFORMATIONS}

Our goal is to define two specific instances of a general transformation that coalesces points in a configuration to form a new configuration, with fewer points and a given mass vector, and ideally with lower potential and standardization maintained.

To begin, we note from Appendix B that it can be shown using a convexity argument that if we partition a discrete configuration $X$ into disjoint subsets of successive point masses and combine each subset into a single point mass at the center of mass of the subset, then we produce a new configuration with fewer point masses and lower potential than that of $X$. Reducing the potential in this fashion is the motivation behind the coalescing transformations.

Given $X=X_{A}$, an $n_{1}$ point configuration, and $B$, an $n_{2}$ point mass vector, where $n_{1}>n_{2}$ and $\operatorname{Min}_{i} b_{i}>\operatorname{Max}_{i} a_{i}$, the ideal coalescing transformation would combine points of $X$ as above and create a new $n_{2}$ point configuration $Z$ having mass vector $B$. Thus $X$ would be transformed from an $n_{1}$ point configuration with mass vector $A$ into an $n_{2}$ point configuration $Z$ with mass vector $B$, and $Z$ would have the same center of mass as $X$ and a lower potential. It is also reasonable to assume, in this case, that the standard deviation would only be altered slightly if $n_{1}$ and $n_{2}$ are large. The ideal, however, is generally not possible, and so our coalescing transformations are approximations to this.

We now give the specific definition of the general form. As in the ideal situation, it takes the $n_{1}$ point configuration $X$ with mass vector $A$ and transforms it into an $n_{2}$ point configuration $Z$ with mass vector $B$. The transformation is easily seen to be well defined provided $\operatorname{Min}_{i} b_{i}>\operatorname{Max}_{i} a_{i}$.

Procedure 7.1 (General Coalescing Transformation). Assuming that $\operatorname{Min}_{i} b_{i}>$ $\operatorname{Max}_{i} a_{i}$, let $s_{i}=\sum_{j=1}^{i} a_{j}, i=1, \ldots, n_{1}$, and $v_{i}=\sum_{j=1}^{i} b_{j}, i=1, \ldots, n_{2}$, denote the partial sums of the mass vectors $A$ and $B$, and set $I_{i}=\left(v_{i-1}, v_{i}\right], i=1, \ldots, n_{2}$, where $v_{0}=0$.

Partition $X$ into $n_{2}$ groups of successive points. A point mass located at $x_{j}$ goes into the $i$ th group if $s_{j} \in I_{i}$. Next, define $W$ to be the configuration obtained by replacing the point masses in each of the groups by the point mass at the center of mass of the group with mass equal to the total mass of the group. Let $R$ denote the mass vector for $W$. The final step is to create $Z$ out of $W$ by simply replacing $R$ by $B$. More precisely, if

$$
W=W_{R}=\left\{w_{1}, w_{2}, \ldots, w_{n_{2}} ; r_{1}, r_{2}, \ldots, r_{n_{2}}\right\},
$$


then

$$
Z=W_{B}=\left\{w_{1}, w_{2}, \ldots, w_{n_{2}} ; b_{1}, b_{2}, \ldots, b_{n_{2}}\right\} .
$$

We note that if the $A$ masses are small relative to the $B$ masses, then the largest $A$ partial sum in $I_{i}$ will be close to $v_{i}$ and the largest in $I_{i-1}$ will be close to $v_{i-1}$. As a result, the total mass placed in the $i$ th group, being the difference of these partial sums, will be close to $v_{i}-v_{i-1}=b_{i}$. Hence, $R \approx B$, and the transformation approximates the ideal.

We now derive properties of this general transformation. We begin with a definition and lemmas dealing with dispersion.

Definition 7.1. The distance between two $n$-point mass vectors $A$ and $B$, denoted $\|A-B\|_{M}$, is defined as:

$$
\|A-B\|_{M}=n \operatorname{Max}_{i}\left|a_{i}-b_{i}\right| .
$$

Lemma 7.1. If $P \in M_{n}[\beta]$, then for $i=1, \ldots, n$,

$$
\frac{1}{n \beta} \leq p_{i} \leq \frac{\beta}{n} \text {, or equivalently, } \frac{1}{n} \leq \beta p_{i}, \quad \text { and } \quad \frac{1}{n} \geq \frac{p_{i}}{\beta} .
$$

Proof. Since $\sum_{i=1}^{n} p_{i}=1, \operatorname{Max}_{i} p_{i} \geq \frac{1}{n}$. Thus, $\beta \geq d(P) \geq \frac{1 / n}{\operatorname{Min}_{i} p_{i}}$. Hence, $\operatorname{Min}_{i} p_{i} \geq \frac{1}{\beta n}$. Also, since $\operatorname{Min}_{i} p_{i} \leq \frac{1}{n}, \beta \geq d(P) \geq \frac{\operatorname{Max}_{i} p_{i}}{1 / n}$. Hence, $\operatorname{Max}_{i} p_{i} \leq \frac{\beta}{n}$.

Lemma 7.2. If $P, Q \in M_{n}[\beta], x_{1}, x_{2}, \ldots, x_{n}$ are given, and $X_{P}=\left\{x_{1}, x_{2}, \ldots, x_{n}\right.$; $\left.p_{1}, p_{2}, \ldots, p_{n}\right\}$ and $X_{Q}=\left\{x_{1}, x_{2}, \ldots, x_{n} ; q_{1}, q_{2}, \ldots, q_{n}\right\}$, then

(i) for $i=1, \ldots, n,\left|p_{i}-q_{i}\right| \leq \beta \operatorname{Min}\left\{p_{i}, q_{i}\right\}\|P-Q\|_{M}$,

(ii) for $i=1, \ldots, n, \frac{1}{\beta^{2}} \leq \frac{q_{i}}{p_{i}} \leq \beta^{2}$,

(iii) for any real-valued function $g$, defined at $x_{1}, x_{2}, \ldots, x_{n},\left|E g\left(X_{P}\right)-E g\left(X_{Q}\right)\right| \leq$ $\beta\|P-Q\|_{M} \operatorname{Min}\left\{E\left|g\left(X_{P}\right)\right|, E\left|g\left(X_{Q}\right)\right|\right\}$,

(iv) $\left|\Pi\left(X_{P}\right)-\Pi\left(X_{Q}\right)\right| \leq\left(\beta+\beta^{3}\right)\|P-Q\|_{M} \operatorname{Min}\left\{\Pi\left(X_{P}\right), \Pi\left(X_{Q}\right)\right\}$.

Proof. (i) From Lemma 7.1, $\frac{1}{n} \leq \beta \operatorname{Min}\left\{p_{i}, q_{i}\right\}$. Thus,

$$
\left|p_{i}-q_{i}\right| \leq \frac{1}{n}\|P-Q\|_{M} \leq \beta \operatorname{Min}\left\{p_{i}, q_{i}\right\}\|P-Q\|_{M} .
$$

(ii) Two applications of Lemma 7.1 yield $q_{i} \leq \frac{\beta}{n} \leq \beta\left(\beta p_{i}\right)$. Reversing the roles of $p_{i}$ and $q_{i}$ gives the other half of the inequality.

(iii) $\left|E g\left(X_{P}\right)-E g\left(X_{Q}\right)\right|=\left|\sum_{i=1}^{n} p_{i} g\left(x_{i}\right)-\sum_{i=1}^{n} q_{i} g\left(x_{i}\right)\right| \leq \sum_{i=1}^{n}\left|p_{i}-q_{i}\right|\left|g\left(x_{i}\right)\right|$ $\leq \sum_{i=1}^{n} \beta q_{i}\|P-Q\|_{M}\left|g\left(x_{i}\right)\right|=\beta E g\left(X_{Q}\right)\|P-Q\|_{M}$. Similarly, $\left|E g\left(X_{P}\right)-E g\left(X_{Q}\right)\right|$ $\leq \beta E g\left(X_{P}\right)\|P-Q\|_{M}$, and the result follows.

(iv) $\log (n)\left|\Pi\left(X_{P}\right)-\Pi\left(X_{Q}\right)\right| \leq \sum_{i<j} \frac{\left|q_{i} q_{j}-p_{i} p_{j}\right|}{x_{j}-x_{i}}=\sum_{i<j} \frac{\left|q_{i} q_{j}-q_{i} p_{j}+q_{i} p_{j}-p_{i} p_{j}\right|}{x_{j}-x_{i}} \leq$ $\sum_{i<j} \frac{q_{i}\left|q_{j}-p_{j}\right|}{x_{j}-x_{i}}+\sum_{i<j} \frac{p_{j}\left|q_{i}-p_{i}\right|}{x_{j}-x_{i}}$.

Using (i) and (ii), it follows that

$$
\begin{array}{r}
\log (n)\left|\Pi\left(X_{P}\right)-\Pi\left(X_{Q}\right)\right| \leq \sum_{i<j} \frac{q_{i} \beta\|P-Q\|_{M} q_{j}}{x_{j}-x_{i}}+\sum_{i<j} \frac{\beta^{2} q_{j} \beta\|P-Q\|_{M} q_{i}}{x_{j}-x_{i}} \\
\quad=\|P-Q\|_{M}\left(\beta+\beta^{3}\right) \sum_{i<j} \frac{q_{i} q_{j}}{x_{j}-x_{i}}=\|P-Q\|_{M}\left(\beta+\beta^{3}\right) \log (n) \Pi\left(X_{Q}\right) .
\end{array}
$$

Interchanging $P$ and $Q$ gives a similar inequality and the result follows. 
The following results are specific to Procedure 7.1. Our first goal is to relate the normalized potential of $X$ and that of its image $Z$ under the general coalescing transformation.

Lemma 7.3. If $A, B, R, n_{1}, n_{2}$ are as given in Procedure 7.1, and if $d(A)$, $d(B) \leq K$, then $\|B-R\|_{M} \leq \frac{n_{2} K}{n_{1}}$.

Proof. Using the notation of Procedure 7.1, let $l_{2}$ denote the largest $A$ partial sum in $I_{i}$, and $l_{1}$ the largest in $I_{i-1}$, and if $i=1$, set $l_{1}=0 . l_{2}$ is at most $v_{i}$, and, from Lemma 7.1, $l_{1} \geq v_{i-1}-\operatorname{Max}_{j} a_{j} \geq v_{i-1}-\frac{K}{n_{1}}$. Thus, $l_{2}-l_{1} \leq v_{i}-\left(v_{i-1}-\frac{K}{n_{1}}\right)=b_{i}+\frac{K}{n_{1}}$. Further, $l_{2} \geq v_{i}-\operatorname{Max}_{j} a_{j}$, and $l_{1} \leq v_{i-1}$. Thus, $l_{2}-l_{1} \geq b_{i}-\frac{K}{n_{1}}$. Finally, since $r_{i}=l_{2}-l_{1}$, we have $\left|r_{i}-b_{i}\right| \leq \frac{K}{n_{1}}$, and hence, $\|R-B\|_{M} \leq \frac{n_{2} K}{n_{1}}$.

Lemma 7.4. If $A, B, R, n_{1}, n_{2}$ are as given in Procedure \%.1, and if $d(A)$, $d(B) \leq K$ and $\frac{n_{1}}{n_{2}}>2 K^{2}$, then $d(R) \leq 3 K^{2}$.

Proof. $\|B-R\|_{M} \leq \frac{n_{2} K}{n_{1}}$ implies $b_{i}-\frac{K}{n_{1}} \leq r_{i} \leq b_{i}+\frac{K}{n_{1}}, i=1,2, \ldots, n_{2}$. Also from Lemma $7.1, \frac{1}{K n_{2}} \leq b_{i} \leq \frac{K}{n_{2}}, i=1,2, \ldots, n_{2}$. Hence, $\frac{1}{K n_{2}}-\frac{K}{n_{1}} \leq r_{i} \leq \frac{K}{n_{2}}+\frac{K}{n_{1}}$, $i=1,2, \ldots, n_{2}$, and thus, $d(R) \leq\left(\frac{K}{n_{2}}+\frac{K}{n_{1}}\right)\left(\frac{1}{K n_{2}}-\frac{K}{n_{1}}\right)^{-1}=\frac{K^{2}\left(\frac{n_{1}}{n_{2}}\right)+K^{2}}{\frac{n_{1}}{n_{2}}-K^{2}}$. For $\frac{n_{1}}{n_{2}}>K^{2}$, this last expression is a decreasing function of $\frac{n_{1}}{n_{2}}$. Thus, if $\frac{n_{1}}{n_{2}}>2 K^{2}$, we find $d(R)$ bounded above by $\frac{K^{2}\left(2 K^{2}\right)+K^{2}}{2 K^{2}-K^{2}} \leq 3 K^{2}$.

The next proposition is the basis for our later showing that two specific versions of the coalescing transformation lead to configurations with normalized potential asymptotically no greater than that of $X$.

Proposition 7.1. If $X, Z, A, B, n_{1}, n_{2}$ are as given in Procedure 7.1 and if $d(A)$, $d(B) \leq K$ and $\frac{n_{1}}{n_{2}}>2 K^{2}$, then

$$
\Pi(Z) \leq\left(1+\frac{n_{2}}{n_{1}}\left(3 K^{3}+27 K^{7}\right)\right) \frac{\log \left(n_{1}\right)}{\log \left(n_{2}\right)} \Pi(X) .
$$

Proof. Using the notation of Procedure 7.1, we have

$$
\frac{\Pi(W)}{\Pi(X)}=\frac{U(W) / \log \left(n_{2}\right)}{U(X) / \log \left(n_{1}\right)} \leq \frac{\log \left(n_{1}\right)}{\log \left(n_{2}\right)} .
$$

Thus, $\Pi(W) \leq \frac{\log \left(n_{1}\right)}{\log \left(n_{2}\right)} \Pi(X)$.

Now, since $W=W_{R}$ and $Z=W_{B}$, on applying Lemma 7.2, part (iv), we have $|\Pi(Z)-\Pi(W)| \leq\left(3 K^{2}+27 K^{6}\right)\|R-B\|_{M} \Pi(W)$, where we've taken $\beta$ equal to $3 K^{2}$ because by Lemma 7.4 both $R$ and $B$ have dispersion less than or equal to $3 K^{2}$. On using Lemma 7.3 , this yields $\Pi(Z) \leq\left(1+\frac{n_{2}}{n_{1}}\left(3 K^{3}+27 K^{7}\right)\right) \Pi(W)$. The result follows from the above inequality relating $\Pi(W)$ and $\Pi(X)$.

Our next goal is to relate the mean and standard deviation of $X$ to that of the image $Z$ under the general coalescing transformation.

Lemma 7.5. If $X, W, A, B, R, n_{1}, n_{2}$ are as given in Procedure 7.1, and if $d(A)$, $d(B) \leq K, \frac{n_{1}}{n_{2}}>2 K^{2}$, and $X$ is contained in the finite interval $[-c, c]$, then

(i) $\mu(X) \stackrel{=}{=} \mu(W)$,

(ii) $|\sigma(X)-\sigma(W)| \leq \frac{2 c K \sqrt{3}}{\sqrt{n_{2}}}$. 
Proof. (i) is an immediate consequence of the construction of $W$.

(ii) Let $G_{j}$ denote the $j$ th group of $X$ mass coordinates whose corresponding masses were combined to yield the mass $r_{j}$ at $w_{j}, x_{t}$ the smallest value in $G_{j}, x_{u}$ the largest, and $\Delta x_{j}=x_{u}-x_{t}$. With $g(x)=x^{2}$ and $w_{j}^{*}$ a point in $\left[x_{t}, x_{u}\right]$ for which $g\left(w_{j}^{*}\right)=\sum_{x_{i} \in G_{j}} \frac{a_{i}}{r_{j}} g\left(x_{i}\right)$, we have

$$
\begin{aligned}
& \left|E X^{2}-E W^{2}\right|=|E g(X)-E g(W)| \\
& \quad \leq \sum_{j=1}^{n_{2}} r_{j}\left|g\left(w_{j}\right)-\sum_{x_{i} \in G_{j}} \frac{a_{i} g\left(x_{i}\right)}{r_{j}}\right|=\sum_{j=1}^{n_{2}} r_{j}\left|g\left(w_{j}\right)-g\left(w_{j}^{*}\right)\right| .
\end{aligned}
$$

Letting $M$ denote the maximum of $|g(x)|$ on $[-c, c]$, and using Lemma 7.1 in conjunction with $d(R) \leq 3 K^{2}$, we find that this last sum is bounded by $M \sum_{j=1}^{n_{2}} r_{j} \Delta x_{j}$ $\leq M \frac{3 K^{2}}{n_{2}} \sum_{j=1}^{n_{2}} \Delta x_{j} \leq M \frac{3 K^{2}}{n_{2}} 2 c=\frac{12 c^{2} K^{2}}{n_{2}}$. Thus,

$$
|\sigma(X)-\sigma(W)| \leq \sqrt{\left|\sigma^{2}(X)-\sigma^{2}(W)\right|}=\sqrt{\left|E X^{2}-E W^{2}\right|} \leq \frac{2 \sqrt{3} c K}{\sqrt{n_{2}}} .
$$

Lemma 7.6. If $X, Z, W, A, B, n_{1}, n_{2}$ are as given in Procedure 7.1, and if $d(A)$, $d(B) \leq K, \frac{n_{1}}{n_{2}}>2 K^{2}$, and $X$ is contained in the finite interval $[-c, c]$, then

(i) $|\mu(Z)-\mu(W)| \leq \frac{3 K^{3} \mathrm{cn}_{2}}{n_{1}}$,

(ii) $|\sigma(Z)-\sigma(W)| \leq 3 \sqrt{3} K^{\frac{3}{2}} c \sqrt{\frac{n_{2}}{n_{1}}}$.

Proof. (i) From Lemma 7.4, it follows that $d(R), d(B) \leq 3 K^{2}$. Applying Lemma 7.2, part (iii), with $g(w)=w, \beta=3 K^{2}$, and $\|R-B\|_{M} \leq \frac{n_{2} K}{n_{1}}$ per Lemma 7.3, we find

$$
|\mu(Z)-\mu(W)|=\left|E W_{B}-E W_{R}\right| \leq 3 K^{2}\left(\frac{n_{2} K}{n_{1}}\right) E W=\frac{3 K^{3} n_{2} E X}{n_{1}} \leq \frac{3 K^{3} n_{2} c}{n_{1}} .
$$

(ii) Applying Lemma 7.2, part (iii) as above, except changing $g(w)$ to $g(w)=w^{2}$, yields

$$
\left|E Z^{2}-E W^{2}\right|=\left|E W_{B}^{2}-E W_{R}^{2}\right| \leq 3 K^{2}\left(\frac{n_{2} K}{n_{1}}\right) E W^{2} \leq \frac{3 K^{3} n_{2} c^{2}}{n_{1}} .
$$

As a result, we have

$$
\begin{aligned}
|\sigma(Z)-\sigma(W)| \leq & \sqrt{\left|\sigma^{2}(Z)-\sigma^{2}(W)\right|} \leq \sqrt{\left|E Z^{2}-E W^{2}\right|} \\
& +\sqrt{|\mu(Z)-\mu(W)|} \sqrt{|\mu(Z)+\mu(W)|} \\
\leq & \sqrt{3} K^{\frac{3}{2}} c \sqrt{\frac{n_{2}}{n_{1}}}+\sqrt{3} K^{\frac{3}{2}} \sqrt{c} \sqrt{\frac{n_{2}}{n_{1}}} \sqrt{2 c} \\
\leq & 3 \sqrt{3} K^{\frac{3}{2}} c \sqrt{\frac{n_{2}}{n_{1}}} .
\end{aligned}
$$

Proposition 7.2. If $X, Z, A, B, n_{1}, n_{2}$ are as given in Procedure 7.1, and if $d(A)$, $d(B) \leq K, \frac{n_{1}}{n_{2}}>2 K^{2}$, and $X$ is contained in the finite interval $[-c, c]$, then

(i) $|\mu(X)-\mu(Z)| \leq \frac{3 K^{3} \mathrm{cn}_{2}}{n_{1}}$,

(ii) $|\sigma(X)-\sigma(Z)| \leq 3 \sqrt{3} K^{\frac{3}{2}} c \sqrt{\frac{n_{2}}{n_{1}}}+\frac{2 \sqrt{3} c K}{\sqrt{n_{2}}}$.

Proof. The results are an immediate consequence of Lemmas 7.5, 7.6. 
Our final goal in developing properties of the general coalescing transformation is to examine the closeness of the mass distribution functions corresponding to $X$ and its image $Z$.

Lemma 7.7. If $X, W, A, B, R, n_{1}, n_{2}$ are as given in Procedure 7.1, and if $d(A)$, $d(B) \leq K, \frac{n_{1}}{n_{2}}>2 K^{2}$, and $F$ and $H$ respectively denote the mass distribution functions corresponding to $X$ and $W$, then $\|F-H\|_{S} \leq \frac{3 K^{2}}{n_{2}}$.

Proof. Let $G_{j}$ denote the $j$ th group of $X$ mass coordinates whose corresponding masses were combined to yield the mass $r_{j}$ at $w_{j}, x_{t}$ the smallest value in $G_{j}$, and $x_{u}$ the largest. Assume that $x \in\left[x_{t}, x_{u}\right]$, and let $x_{t}^{-}$denote an $x$ value less than $x_{t}$ but arbitrarily close to $x_{t}$. Then $F\left(x_{t}^{-}\right) \leq F(x) \leq F\left(x_{u}\right)$ and $F\left(x_{t}^{-}\right) \leq H(x) \leq F\left(x_{u}\right)$. Thus, $|F(x)-H(x)| \leq F\left(x_{u}\right)-F\left(x_{t}^{-}\right)=r_{j} \leq \frac{3 K^{2}}{n_{2}}$, with the last inequality following from Lemma 7.1 in conjunction with $d(R) \leq 3 K^{2}$. Now if $x \notin\left[x_{t}, x_{u}\right]$ for any $j$, then $H(x)=F(x)$, and so $\|F-H\|_{S} \leq \frac{3 K^{2}}{n_{2}}$.

Lemma 7.8. If $W, Z, A, B, R, n_{1}, n_{2}$ are as given in Procedure 7.1, and if $d(A)$, $d(B) \leq K, \frac{n_{1}}{n_{2}}>2 K^{2}$, and $H$ and $G$ respectively denote the mass distribution functions corresponding to $W$ and $Z$, then $\|G-H\|_{S} \leq \frac{3 K^{3} n_{2}}{n_{1}}$.

Proof. If $w \geq w_{n_{2}}$, then $H(w)=G(w)=1$, and if $w<w_{1}, H(w)=G(w)=0$. For $w \in\left[w_{i-1}, w_{i}\right), i=2, \ldots, n_{2}, H(w)=H\left(w_{i-1}\right)$ and $G(w)=G\left(w_{i-1}\right)$. Thus, $\|H-G\|_{S}=\operatorname{Max}_{1 \leq i \leq n_{2}}\left|H\left(w_{i}\right)-G\left(w_{i}\right)\right|=\operatorname{Max}_{1 \leq i \leq n_{2}}\left|\sum_{j=1}^{i} r_{j}-\sum_{j=1}^{i} b_{j}\right| \leq$ $\sum_{j=1}^{n_{2}}\left|r_{j}-b_{j}\right|$.

Using $d(R), d(B) \leq 3 K^{2}$, Lemma 7.2, part (i) and Lemma 7.3, we find the last sum bounded by $\sum_{j=1}^{n_{2}} 3 K^{2}\|R-B\|_{M} r_{j}=3 K^{2}\|R-B\|_{M} \leq 3 K^{2} \frac{n_{2} K}{n_{1}}=\frac{3 n_{2} K^{3}}{n_{1}}$.

Proposition 7.3. If $X, Z, A, B, n_{1}, n_{2}$ are as given in Procedure 7.1, and if $d(A)$, $d(B) \leq K, \frac{n_{1}}{n_{2}}>2 K^{2}$, and $F$ and $G$ respectively denote the mass distribution functions corresponding to $X$ and $Z$, then $\|F-G\|_{S} \leq \frac{3 K^{2}}{n_{2}}+\frac{3 K^{3} n_{2}}{n_{1}}$.

Proof. The result is an immediate consequence of Lemmas 7.7, 7.8.

We now define two specific forms of the general coalescing transformation. In the definitions, $[c]$ denotes the greatest integer less than or equal to $c$.

Definition 7.2. The coalescing-to-equal-mass transformation, denoted by $K_{T}$, is defined by Procedure 7.1 with $X$ a given $n_{1}=n$ point configuration and $B$ the $n_{2}=\left[\frac{n}{\log (n)}\right]$ point mass vector with all masses equal. $Z$, the image of $X$ under $K_{T}$, is denoted as $K_{T}(X)$.

In this form of the coalescing transformation, $n$, the number of points in $X$, determines the number of equal masses in $B$.

Definition 7.3. The coalescing-from-equal-mass transformation, denoted by $K_{F}$, is defined by Procedure 7.1 with $B$ a given $n_{2}=n$ point mass vector and $X$ any given configuration having $n_{1}=[n \log (n)]+1$ equal masses. $Z$, the image of $X$ under $K_{F}$, is denoted as $K_{F}(X: B)$.

In this form of the coalescing transformation, the number of equal-mass points in $X$ is determined by $n$, the number of masses in $B$. 
Proposition 7.4. With $n$ as given in Definitions 8.2, 8.3,

(i) $K_{T}$ is well defined provided $n>e^{d(A)}$.

(ii) $K_{F}$ is well defined provided $n>e^{d(B)}$.

Proof. Using the notation of Procedure $7.1, K_{T}$ and $K_{F}$ will be well defined provided that $\operatorname{Min}_{i} b_{i}>\operatorname{Max}_{i} a_{i}$. In the case of $K_{T}, \operatorname{Min}_{i} b_{i}=\frac{1}{\left[\frac{n}{\log (n)}\right]}$, and Lemma 7.1 gives $\operatorname{Max}_{i} a_{i} \leq \frac{d(A)}{n}$. It follows that $\frac{\operatorname{Min}_{i} b_{i}}{\operatorname{Max}_{i} a} \geq\left(\frac{n}{d(A)}\right)\left(\frac{1}{\left[\frac{n}{\log (n)}\right]}\right) \geq\left(\frac{n}{d(A)}\right)\left(\frac{\log (n)}{n}\right)=$ $\frac{\log (n)}{d(A)}>1$ when $n>e^{d(A)}$.

In the case of $K_{F}, \operatorname{Max}_{i} a_{i}=\frac{1}{[n \log (n)]+1}$ and Lemma 7.1 gives $\operatorname{Min}_{i} b_{i} \geq \frac{1}{d(B) n}$. It follows that $\frac{\operatorname{Min}_{i} b_{i}}{\operatorname{Max}_{i} a} \geq\left(\frac{[n \log (n)]+1}{n d(B)}\right) \geq \frac{\log (n)}{d(B)}>1$ when $n>e^{d(B)}$.

The final two propositions in this section contain the results that we will subsequently use to establish uniform convergence of the mass distribution functions. Their implication is that the transformations $K_{T}$ and $K_{F}$, in acting on standardized uniformly bounded configurations having bounded normalized potential and mass vectors of bounded dispersion, asymptotically produce configurations with no greater normalized potential and with standardization and mass distribution maintained.

Notation 7.1. For a sequence $\left\{\alpha_{i}\right\}, \overline{\operatorname{Lim}} \alpha_{i}$ will denote $\operatorname{LimSup}{ }_{i \rightarrow \infty} \alpha_{i}$, and $\underline{\operatorname{Lim}} \alpha_{i}$ will denote $\operatorname{LimInf}_{i \rightarrow \infty} \alpha_{i}$.

Proposition 7.5. Let $\left\{X_{i}\right\}_{i=1}^{\infty}$ be a sequence of standardized $l_{i}$-point configurations for which $\left\{\Pi\left(X_{i}\right)\right\}_{i=1}^{\infty}$ is bounded, each configuration has a mass vector with dispersion less than or equal to $K$, and each is contained in a given finite interval $[-c, c]$. Then, if $l_{i}>e^{K}, l_{i} \rightarrow \infty, Z_{i}=K_{T}\left(X_{i}\right)$, and $F_{i}$ and $G_{i}$ are respectively the mass distribution functions for $X_{i}$ and $Z_{i}$,

(i) $\overline{\operatorname{Lim}} \Pi\left(Z_{i}\right) \leq \overline{\operatorname{Lim}} \Pi\left(X_{i}\right)$.

(ii) $\operatorname{Lim} \mu\left(Z_{i}\right)=0$.

(iii) $\operatorname{Lim} \sigma\left(Z_{i}\right)=1$.

(iv) $\operatorname{Lim}\left\|F_{i}-G_{i}\right\|_{S}=0$.

(v) Each member of the sequence $\left\{Z_{i}\right\}_{i=1}^{\infty}$ is contained in $[-c, c]$.

Proof. (i) We can apply Proposition 7.1. Using the notation of Definition 7.2, with $n_{1}=l_{i}$ and $n_{2}=\left[\frac{l_{i}}{\log \left(l_{i}\right)}\right]$, we see that $\frac{n_{2}}{n_{1}} \rightarrow 0$ and $\frac{\log \left(n_{1}\right)}{\log \left(n_{2}\right)} \rightarrow 1$. We take $A$ equal to the mass vector for $X_{i}$ and $B$ the equal-mass vector. Each has dispersion less than or equal to $K$. Applying the proposition with $X=X_{i}$ and $Z=Z_{i}$ yields the result.

(ii) Proceeding as in (i), we can apply Proposition 7.2. The result follows since $\mu\left(X_{i}\right)=0$ and $\frac{n_{2}}{n_{1}} \rightarrow 0$.

(iii) As in (ii), the proof uses Proposition 7.2. This time in conjunction with $\sigma\left(X_{i}\right)=1, \frac{n_{2}}{n_{1}} \rightarrow 0$, and $n_{2} \rightarrow \infty$.

(iv) This follows from Proposition 7.3.

(v) This follows immediately from Procedure 7.1, which defines the general coalescing transformation.

Proposition 7.6. Let $\left\{B_{i}\right\}_{i=1}^{\infty}$ be a sequence of $l_{i}$-point mass vectors for which $d\left(B_{i}\right) \leq K$ and $\left\{X_{i}\right\}_{i=1}^{\infty}$ be a sequence of standardized $\left[l_{i} \log \left(l_{i}\right)\right]+1$ point equalmass configurations for which $\left\{\Pi\left(X_{i}\right)\right\}_{i=1}^{\infty}$ is bounded and each configuration is contained in a given finite interval $[-c, c]$. Then, if $l_{i}>e^{K}, l_{i} \rightarrow \infty, Z_{i}=$ $K_{F}\left(X_{i}: B_{i}\right)$, and $F_{i}$ and $G_{i}$ are respectively the mass distribution functions for $X_{i}$ and $Z_{i}$, 
(i) $\overline{\operatorname{Lim}} \Pi\left(Z_{i}\right) \leq \overline{\operatorname{Lim}} \Pi\left(X_{i}\right)$.

(ii) $\operatorname{Lim} \mu\left(Z_{i}\right)=0$.

(iii) $\operatorname{Lim} \sigma\left(Z_{i}\right)=1$.

(iv) $\operatorname{Lim}\left\|F_{i}-G_{i}\right\|_{S}=0$.

Proof. (i) We can apply Proposition 7.1. Using the notation of Definition 7.3, with $n_{2}=l_{i}$ and $n_{1}=\left[l_{i} \log \left(l_{i}\right)\right]+1$, we see that $\frac{n_{2}}{n_{1}} \rightarrow 0$ and $\frac{\log \left(n_{1}\right)}{\log \left(n_{2}\right)} \rightarrow 1$. We take $A$ to be the $n_{1}$-point equal-mass vector and $B$ the mass vector for $X_{i}$. Each has dispersion less than or equal to $K$. Applying the proposition with $X=X_{i}$ and $Z=Z_{i}$ yields the result. 7.3 .

(ii)-(iv) Proceeding as in (i), these follow respectively from Propositions 7.2 and

\section{THE HISTOGRAM TRANSFORMATION}

In this section, we will define a histogram-like continuous mass configuration, $H(X)$, corresponding to a discrete $n$-point equal-mass configuration $X$.

Each bar in the histogram will correspond to a grouping of adjacent point masses. For large $n$, we will want many classes (groupings), many points in each class, and simultaneously, as $n \rightarrow \infty$, total mass in each class to go to 0 in a rather precise fashion.

Let $X=\left\{x_{1}, x_{2}, \ldots, x_{n} ; \frac{1}{n}, \frac{1}{n}, \ldots, \frac{1}{n}\right\}$ be an $n$-point equal-mass configuration, where, to avoid picky details, we assume $n \geq 100$. $H(X)$ will be determined by a density function $h(x)$ which is constructed as follows:

Set $\delta(n)=n^{-\left(\frac{1}{\log \log (n)}\right)}$. The number of class intervals is denoted by $N$, where $N=\left[\frac{1}{\delta(n)}\right]$, and the intervals are given by $\left[x_{i_{0}}, x_{i_{1}}\right),\left[x_{i_{1}}, x_{i_{2}}\right), \ldots,\left[x_{i_{N-2}}, x_{i_{N-1}}\right)$, $\left[x_{i_{N-1}}, x_{i_{N}}\right]$, where $i_{0}=1, i_{N}=n$, and $i_{j}=[j n \delta(n)], j=1, \ldots, N-1 . \Delta x_{j}=$ $x_{i_{j}}-x_{i_{j-1}}$ denote the length of the $j$ th class interval.

The number of points in the $j$ th class interval is denoted by $n_{j}$ where $n_{j}=$ $i_{j}-i_{j-1}, j=1, \ldots, N-1$, and $n_{N}=i_{N}-i_{N-1}+1$, and the total mass in the interval is denoted by $\Delta m_{j}=\frac{n_{j}}{n}$. $h(x)$ is defined by

$$
h(x)=\left\{\begin{array}{l}
0: x<x_{1} \\
\frac{\Delta m_{j}}{\Delta x_{j}}: x \text { in the } j \text { th class interval. } \\
0: x>x_{n}
\end{array}\right.
$$

It is easily seen that $\delta(n) \rightarrow 0$ as $n \rightarrow \infty$. Further,

$$
\int_{-\infty}^{\infty} h(x) d x=\sum_{i=1}^{N}\left(\frac{\Delta m_{i}}{\Delta_{i}}\right) \Delta x_{i}=1 .
$$

In the next two lemmas we establish some additional properties for $h(x)$.

Table 8.1 gives a feel for $h(x)$ via some selected values of the parameters.

TABle 8.1. Selected Histogram Parameters

\begin{tabular}{clrrlrl}
$n$ & $\delta(n)$ & \multicolumn{1}{c}{$N$} & \multicolumn{1}{c}{$n_{1}$} & $\Delta m_{1}$ & \multicolumn{1}{c}{$n_{N}$} & $\Delta m_{N}$ \\
\hline $10^{2}$ & .049 & 20 & 3 & .03 & 8 & .08 \\
$10^{3}$ & .028 & 35 & 27 & .027 & 48 & .048 \\
$10^{6}$ & .00518 & 192 & 5186 & .005186 & 9150 & .00915
\end{tabular}


Lemma 8.1. If $n \geq 100$, then for $j=1, \ldots, N$,

(i) $\frac{n \delta(n)}{2} \leq n_{j} \leq 3 n \delta(n)$.

(ii) $\frac{\delta(n)}{2} \leq \Delta m_{j} \leq 3 \delta(n)$.

Proof. (i) It can be shown that $n \delta(n)$ is an increasing function of $n$ for $n>e$, and hence, for $n \geq 100, n \delta(n) \geq 100 \delta(100)>4$.

To prove the result for $j=1$, we use $n_{1}=[n \delta(n)]-1$ together with $\frac{n \delta(n)}{2} \leq$ $n \delta(n)-2 \leq[n \delta(n)]-1 \leq n \delta(n)$.

For $j=2, \ldots, N-1$,

$n_{j}=[j n \delta(n)]-[(j-1) n \delta(n)] \leq j n \delta(n)-(j-1) n \delta(n)+1=n \delta(n)+1 \leq 2 n \delta(n)$.

Further, $n_{j} \geq j n \delta(n)-1-(j-1) n \delta(n)=n \delta(n)-1 \geq \frac{n \delta(n)}{2}$.

For $j=N$,

$$
\begin{aligned}
n_{N} & =n-[(N-1) n \delta(n)]+1 \leq n-(N-1) n \delta(n)+2 \\
& \leq(n+2)+n \delta(n)-\left(\frac{1}{\delta(n)}-1\right) n \delta(n)=2 n \delta(n)+2 \leq 3 n \delta(n),
\end{aligned}
$$

and

$$
\begin{aligned}
n_{N} & =n-[(N-1) n \delta(n)]+1 \geq n+1-(N-1) n \delta(n) \\
& \geq n \delta(n)+(n+1)-\frac{1}{\delta(n)} n \delta(n) \geq \frac{n \delta(n)}{2} .
\end{aligned}
$$

(ii) This follows immediately from (i).

Lemma 8.2. Given $\varepsilon>0$ and $c>0$, there exists an integer $L>0$, such that if $n>L$, then, for $j=1,2, \ldots, N$,

(i) $\frac{\left|\log \left(\Delta m_{j}\right)\right|}{\log (n)}<\varepsilon$,

(ii) $\Delta m_{j}<\varepsilon$,

(iii) $n_{j}>c$.

Proof. The results are implied by inequalities stemming from the previous lemma as follows:

(i) $\frac{\left|\log \left(\Delta m_{j}\right)\right|}{\log (n)} \leq \frac{\left|\log \left(\frac{\delta(N)}{2}\right)\right|}{\log (n)}=\frac{1}{\log \log (n)}+\frac{\log (2)}{\log (n)}$.

(ii) $\log \left(\Delta m_{j}\right) \leq \log (3 \delta(n))=\log (3)-\frac{\log (n)}{\log \log (n)}$.

(iii) $\log \left(n_{j}\right)>\log (n)+\log (\delta(n))-\log (2)=\log (n)\left(1-\frac{1}{\log \log (n)}\right)-\log (2)$.

The next lemma begins the analysis of the relationship between the normalized potentials of $X$ and $H(X)$.

Lemma 8.3. Let $R>0$ be given and $y_{1}, y_{2}, \ldots, y_{l}$ be $l$ positive real numbers satisfying $\sum_{i=1}^{l} y_{i}=R$; then $\sum_{i=1}^{l} \frac{1}{y_{i}} \geq \frac{l^{2}}{R}$.

Proof. Let $G\left(y_{1}, y_{2}, \ldots, y_{l}\right)=\sum_{i=1}^{l} \frac{1}{y_{i}}$. Using the auxiliary function $F=G-$ $\lambda\left(\sum_{i=1}^{l} y_{i}-R\right)$ and applying the method of Lagrange multipliers, we find that $\nabla F=0$ implies $-\frac{1}{y_{i}^{2}}=\lambda, i=1,2, \ldots, l$. Thus, at the minimum value of $G$ all the $y_{i}$ are equal. Their value is $\frac{R}{l}$, and the minimum value of $G$ equals $\frac{l^{2}}{R}$.

In the next proposition the configuration $X$ is not restricted to having a total mass of 1 . The total mass can be any positive number. 
Proposition 8.1. Given $\varepsilon>0$, there exists a number $c>0$, such that for $k>c$, any $k$-point equal-mass configuration $X$ has the property that $\Pi(X)>\frac{(1-\varepsilon) T^{2}}{\left(x_{k}-x_{1}\right)}$ where $T$ is the total mass of $X$.

Proof. Let $X=\left\{x_{1}, x_{2}, \ldots, x_{k} ; \frac{T}{k}, \frac{T}{k}, \ldots, \frac{T}{k}\right\}$ be a $k$-point equal-mass configuration with total mass $T$. Set $r_{i}=x_{i+1}-x_{i}, i=1, \ldots, k-1$, and let

$$
\begin{gathered}
S_{1}=\left(\frac{1}{r_{1}}+\frac{1}{r_{2}}+\cdots+\frac{1}{r_{k-1}}\right), \quad S_{2}=\left(\frac{1}{r_{1}+r_{2}}+\frac{1}{r_{2}+r_{3}}+\cdots+\frac{1}{r_{k-2}+r_{k-1}}\right) \\
S_{3}=\left(\frac{1}{r_{1}+r_{2}+r_{3}}+\frac{1}{r_{2}+r_{3}+r_{4}}+\cdots+\frac{1}{r_{k-3}+r_{k-2}+r_{k-1}}\right), \ldots \\
S_{k-1}=\left(\frac{1}{r_{1}+r_{2}+\cdots+r_{k-1}}\right) .
\end{gathered}
$$

$\Pi(X)$ can be written as $\Pi(X)=\frac{T^{2}}{k^{2} \log (k)} \sum_{j=1}^{k-1} S_{j}$.

Now in $S_{j}$, the sum of the $k-j$ denominators is less than or equal to $j\left(x_{k}-x_{1}\right)$. Applying Lemma 8.3 to $S_{j}$, with $l=k-j$ and $R \leq j\left(x_{k}-x_{1}\right)$, we find $S_{j} \geq \frac{(k-j)^{2}}{j\left(x_{k}-x_{1}\right)}$. As a result,

$$
\begin{aligned}
\Pi(X) & \geq \frac{T^{2}}{k^{2}\left(x_{k}-x_{1}\right) \log (k)} \sum_{j=1}^{k} \frac{(k-j)^{2}}{j} \\
& \geq \frac{T^{2}}{k^{2}\left(x_{k}-x_{1}\right) \log (k)}\left(\sum_{j=1}^{k} \frac{k^{2}}{j}-\sum_{j=1}^{k} 2 k\right) .
\end{aligned}
$$

Noting that $\sum_{j=1}^{k} \frac{1}{j}=\log (k)+\gamma+\varepsilon_{1}(k)$, where $\gamma$ is Euler's gamma and $\varepsilon_{1}(k) \rightarrow 0$ as $k \rightarrow \infty$, we find $\Pi(X) \geq \frac{T^{2}}{\left(x_{k}-x_{1}\right)}\left(1+\frac{\gamma+\varepsilon_{1}(k)-2}{\log (k)}\right)$. Choosing $c$, such that $k>c$ implies $\left|\frac{\gamma+\varepsilon_{1}(k)-2}{\log (k)}\right|<\varepsilon$, yields the result.

Lemma 8.4. Given $\varepsilon>0$, there exists an integer $N_{1}$, such that if $X$ is any equalmass configuration with more than $N_{1}$ points, then its normalized potential and that of $H(X)$ satisfy $\Pi(X) \geq(1-\varepsilon) \Pi(H(X))$.

Proof. Let $X$ be an $n$-point equal-mass configuration where $n>100$. Using the notation employed in defining $H(X)$, let $Y_{j}$ denote the configuration of point masses from $X$ which are contained in the $j$ th class interval, $j=1, \ldots, N$. Pick $\varepsilon_{1}>0$ such that $\varepsilon_{1}<1$ and $\left(1-\varepsilon_{1}\right)^{2} \geq 1-\varepsilon$.

On noting that $Y_{j}$ has total mass $\Delta m_{j}$, contains $n_{j}$ points, and has distance between its extreme points less than or equal to $\Delta x_{j}$, we find from Proposition 8.1 that there is a $c>0$, such that if $n_{j}>c$, then $\Pi\left(Y_{j}\right) \geq \frac{\left(1-\varepsilon_{1}\right)\left(\Delta m_{j}\right)^{2}}{\Delta x_{j}}$. In conjunction with this, using Lemma 8.2, we can find an integer $N_{1}$ such that if $n>N_{1}$, then $n_{j}>c$ and $\left|\frac{\log \left(\Delta m_{j}\right)}{\log (n)}\right|<\varepsilon_{1}, j=1, \ldots, N$.

Let $n$ be greater than $N_{1}$. Using the fact that $U(X) \geq \sum_{j=1}^{N} U\left(Y_{j}\right)$, we have 


$$
\begin{aligned}
\Pi(X) & \geq \frac{1}{\log (n)} \sum_{j=1}^{N} U\left(Y_{j}\right)=\frac{1}{\log (n)} \sum_{j=1}^{N}\left(\log \left(n_{j}\right) \Pi\left(Y_{j}\right)\right) \\
& =\frac{1}{\log (n)} \sum_{j=1}^{N}\left(\log \left(\frac{n_{j}}{n}\right)+\log (n)\right) \Pi\left(Y_{j}\right)=\sum_{j=1}^{N}\left(1+\frac{\log \left(\Delta m_{j}\right)}{\log (n)}\right) \Pi\left(Y_{J}\right) \\
& \geq \sum_{j=1}^{N}\left(1-\varepsilon_{1}\right) \Pi\left(Y_{j}\right) \geq\left(1-\varepsilon_{1}\right)^{2} \sum_{j=1}^{N}\left(\frac{\Delta m_{j}}{\Delta x_{j}}\right)^{2} \Delta x_{j} \\
& =\left(1-\varepsilon_{1}\right)^{2} \int_{-\infty}^{\infty} h^{2}(x) d x \geq(1-\varepsilon) \Pi(H(X)) .
\end{aligned}
$$

The next lemma indicates how well $H$ maintains standardization.

Lemma 8.5. If $X$ is a standardized n-point equal-mass configuration which is contained in a finite interval $[-c, c]$, and for which $n>100$, then

(i) $|\mu(H(X))| \leq 6 c \delta(n)$.

(ii) $\left|\sigma^{2}(H(X))-1\right| \leq 12 \delta(n) c^{2}(1+3 \delta(n))$.

Proof. Let $g$ be a continuously differentiable function defined on $[-c, c], I_{j}$ the $j$ th class interval in the construction of $H(X), \bar{I}_{j}$ the closure of $I_{j}, B_{j}$ the maximum of $g(x)$ on $\bar{I}_{j}$, and $b_{j}$ the minimum of $g(x)$ on $\bar{I}_{j}$.

Using the notation involved in the definition of $H(X)$, we have $E g(X)=$ $\sum_{j=1}^{N} \sum_{x_{i} \in I_{j}} \frac{1}{n} g\left(x_{i}\right)$ and

$$
E g(H(X))=\sum_{j=1}^{N} \int_{I_{j}} g(x) h(x) d x=\sum_{j=1}^{N}\left(\frac{\Delta m_{j}}{\Delta x_{j}}\right) \int_{I_{j}} g(x) d x .
$$

From the above expressions it follows that both

$$
\sum_{j=1}^{N} b_{j} \Delta m_{j} \leq E g(X) \leq \sum_{j=1}^{N} B_{j} \Delta m_{j}
$$

and

$$
\sum_{j=1}^{N} b_{j} \Delta m_{j} \leq E g(H(X)) \leq \sum_{j=1}^{N} B_{j} \Delta m_{j}
$$

Letting $Q=\operatorname{Max}\left[\left|g^{\prime}(x)\right|: x \in[-c, c]\right]$ and employing Lemma 8.1, we find

$$
|E g(H(X))-E g(X)| \leq \sum_{j=1}^{N}\left(B_{j}-b_{j}\right) \Delta m_{j} \leq Q \sum_{j=1}^{N} \Delta x_{j} \Delta m_{j} \leq 3 \delta(n)(2 c) Q .
$$

Taking $g(x)=x$ gives $Q=1$ and yields $|\mu(H(X))| \leq 6 c \delta(n)$. On letting $g(x)=x^{2}$, we get $Q=2 c$ and $\left|\sigma^{2}(H(X))-1\right|=\left|E(H(X))^{2}-\mu^{2}(H(X))-E X^{2}\right| \leq$ $\left|E(H(X))^{2}-E X^{2}\right|+\mu^{2}(H(X)) \leq(2 c)(6 c) \delta(n)+36 c^{2} \delta^{2}(n)$.

The following lemma indicates how well $H$ maintains mass distribution.

Lemma 8.6. If $F$ and $G$ respectively denote the mass distribution functions corresponding to $X$ and $H(X)$, then $\|F-G\|_{S} \leq 3 \delta(n)$. 
Proof. If $x<x_{1}$ or $x>x_{n}$, then $|F(x)-G(x)|=0$. Assume $x$ is contained in $I_{j}$, the $j$ th class interval. Let $a$ denote the left endpoint of this interval and set $L=\operatorname{Lim}_{x \rightarrow a^{-}} F(x)=\operatorname{Lim}_{x \rightarrow a^{-}} G(x)$. Both $F(x)$ and $G(x)$ are bounded below by $L$ and above by $L+\Delta m_{j}$. As a result, using Lemma 8.1, $|F(x)-G(x)| \leq 3 \delta(n)$.

The results that we will need to establish uniform convergence are given in the next proposition. It is analogous to Propositions 7.5, 7.6. Its implication is that $H$, when acting on uniformly bounded standardized equal-mass configurations having bounded normalized potential, asymptotically produces configurations with no greater normalized potential and with standardization and mass distribution maintained.

Proposition 8.2. Let $\left\{X_{i}\right\}_{i=1}^{\infty}$ be a sequence of standardized $l_{i}$-point equal-mass configurations, each of which is contained in a given finite interval $[-c, c]$ and for which the sequence $\left\{\Pi\left(X_{i}\right)\right\}_{i=1}^{\infty}$ is bounded. Then, if $l_{i}>100, l_{i} \rightarrow \infty, Z_{i}=H\left(X_{i}\right)$, and $F_{i}$ and $G_{i}$ are respectively the mass distribution functions for $X_{i}$ and $Z_{i}$,

(i) $\overline{\operatorname{Lim}} \Pi\left(Z_{i}\right) \leq \overline{\operatorname{Lim}} \Pi\left(X_{i}\right)$.

(ii) $\operatorname{Lim} \mu\left(Z_{i}\right)=0$.

(iii) $\operatorname{Lim} \sigma\left(Z_{i}\right)=1$.

(iv) $\operatorname{Lim}\left\|F_{i}-G_{i}\right\|_{S}=0$.

(v) Each member of the sequence $\left\{Z_{i}\right\}_{i-1}^{\infty}$ is contained in $[-c, c]$.

Proof. (i) This follows from Lemma 8.4.

(ii)-(iv) These follow from Lemmas 8.5, 8.6 using the fact that $\delta\left(l_{i}\right) \rightarrow 0$ as $i \rightarrow \infty$.

(v) This follows from the fact that for a discrete configuration $X, h(x)$, the density function determining $H(X)$, is constructed in such a manner as to be 0 outside of any interval which contains $X$.

\section{The CONTINUOUS-TO-DISCRETE TRANSFORMATION}

This transformation plays the opposite role from that of the histogram transformation. It takes a continuous configuration and produces an equal-mass discrete configuration.

Definition 9.1. Let $X$ be a continuous configuration with distribution function $F$. The n-point continuous-to-discrete transformation acting on $X$ is denoted by $C_{D}(X ; n)$. It produces an $n$-point equal-mass configuration defined as $C_{D}(X ; n)=$ $\left(F^{-1}(.5 / n), F^{-1}(1.5 / n), \ldots, F^{-1}((n-.5) / n) ; \frac{1}{n}, \frac{1}{n}, \ldots, \frac{1}{n}\right)$, where, for $y>0$, $F^{-1}(y)=\operatorname{Inf}[x: F(x)=y]$.

Our objectives are similar to those for the other transformations. We will show under regularity conditions that in acting on a bounded standardized configuration $X$, asymptotically as $n \rightarrow \infty, C_{D}$ maintains standardization and does not increase normalized potential. For this transformation, we are not interested in the effects on mass distribution.

Lemma 9.1. Let $F(x)$ be continuous, have a continuous positive first derivative on $[a, b]$, and a continuous second derivative on $[a, b]$. Then, if $u$ and $v$ are distinct points in the interval $[F(a), F(b)], \frac{1}{F^{-1}(u)-F^{-1}(v)}=\frac{F^{\prime}\left(F^{-1}(u)\right)}{u-v}+b(u, v)$, where $b(u, v)$ is bounded independent of $u$ and $v$. 
Proof. Let $H(y)=F^{-1}(y)$, and with $F^{\prime}\left(F^{-1}(u)\right)=\frac{1}{H^{\prime}(u)}$, set $b(u, v)=\frac{1}{H(u)-H(v)}-$ $\frac{1 / H^{\prime}(u)}{u-v}$. Taylor's formula with remainder yields $H(u)-H(v)=(u-v) H^{\prime}(u)-$ $\frac{(u-v)^{2}}{2} H^{\prime \prime}(\xi)$ where $\xi$ is between $u$ and $v$. On substituting this in the expression for $b$ and doing some manipulation, we find $b(u, v)=\frac{1}{2}\left(\frac{H^{\prime \prime}(\xi) /\left(H^{\prime}(u)\right)^{2}}{1-(1 / 2)(u-v)\left(H^{\prime \prime}(\xi) / H^{\prime}(u)\right)}\right)$.

On using $H^{\prime \prime}(y)=-\frac{F^{\prime \prime}\left(F^{-1}(y)\right)}{\left(F^{\prime}\left(F^{-1}(y)\right)\right)^{3}}$ and letting $f(x)=F^{\prime}(x), x_{u}=F^{-1}(u)$, and $\phi=F^{-1}(\xi)$, we have $b(u, v)=(1 / 2) \frac{-f^{\prime}(\phi) f^{2}\left(x_{u}\right) / f^{3}(\phi)}{1+(1 / 2)(u-v)\left(f\left(x_{u}\right) f^{\prime}(\phi) / f^{3}(\phi)\right)}$, where $x_{u}, \phi \in$ $[a, b]$ and both $-f^{\prime}(\phi) f^{2}\left(x_{u}\right) / f^{3}(\phi)$ and $\left.f\left(x_{u}\right) f^{\prime}(\phi) / f^{3}(\phi)\right)$ are bounded independent of $u$ and $v$ in $[F(a), F(b)]$.

Thus, if in $[F(a), F(b)] \times\{F(a), F(b)]$ there exists a sequence $\left\{\left(u_{i}, v_{i}\right)\right\}$ for which $\left|b\left(u_{i}, v_{i}\right)\right| \rightarrow \infty$, it follows that $\left|u_{i}-v_{i}\right|$ is bounded away from 0 . So, if such a sequence exists, with no loss, it can be assumed that $u_{i} \rightarrow u^{*}$ and $v_{i} \rightarrow v^{*}$, where $u^{*} \neq v^{*}$. But $b(u, v)=\frac{1}{F^{-1}(u)-F^{-1}(v)}-\frac{f\left(F^{-1}(u)\right)}{u-v}$ is a continuous function of $(u, v)$ at $\left(u^{*}, v^{*}\right)$, and thus it is not possible for $\left|b\left(u_{i}, v_{i}\right)\right|$ to approach infinity. We conclude that no such sequence exists, and it follows that $b(u, v)$ is bounded independent of $u$ and $v$ in $[F(a), F(b)]$.

Definition 9.2. Let $Z=Z_{A}$ and $W=W_{B}$ be disjoint configurations with no restriction on the total mass of either. The cross potential, denoted $C(Z, W)$, is defined as $C(Z, W)=\sum_{i, j} \frac{a_{i} b_{j}}{\left|z_{i}-w_{j}\right|}$.

Proposition 9.1. Let $X$ be a continuous standardized configuration contained in the finite interval $[c, d]$. Let $F$ denote its mass distribution function and assume the following conditions hold: $F$ has continuous first and second derivatives on $[c, d]$, $F^{\prime}(x)>0$ on $(c, d), F(c)=0$, and $F(d)=1$. Then as $n \rightarrow \infty$,

(i) $\operatorname{Lim} \mu\left(C_{D}(X ; n)\right)=0$ and $\operatorname{Lim} \sigma\left(C_{D}(X ; n)\right)=1$,

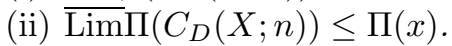

Proof. For convenience, the symbol " " attached as a superscript to an integer $j$ will denote $j-.5$, i.e. $j^{\sim}=j-.5$.

(i) $\mu\left(C_{D}(X ; n)\right)=\frac{1}{n} \sum_{i=1}^{n} F^{-1}\left(i^{\sim} / n\right)$. This is a Riemann sum and tends to $\int_{0}^{1} F^{-1}(y) d y=\int_{c}^{d} x f(x) d x=\mu(X)=0$, where $f(x)=F^{\prime}(x)$ is the mass density function. The second part follows from the fact that

$$
E\left(C_{D}(X ; n)\right)^{2}=\frac{1}{n} \sum_{i=1}^{n}\left(F^{-1}\left(i^{\sim} / n\right)\right)^{2}
$$

tends to $\int_{0}^{1}\left(F^{-1}(y)\right)^{2} d y=\int_{c}^{d} x^{2} f(x) d x=1$.

(ii) Given $\varepsilon>0$, partition $C_{D}(X ; n)$ into three disjoint configurations $Y_{1}, Y_{2}, Y_{3}$ as follows: $Y_{1}$ will be those point masses in the interval $\left[c, F^{-1}(\varepsilon)\right], Y_{2}$ those in $\left(F^{-1}(\varepsilon), F^{-1}(1-\varepsilon)\right)$, and $Y_{3}$ those in $\left[F^{-1}(1-\varepsilon), d\right]$. (These configurations generally do not have total mass equal to 1 .)

Using this partition, the potential of $C_{D}(X ; n)$ can be written $U\left(C_{D}(X ; n)\right)=$ $\sum_{i=1}^{3} U\left(Y_{i}\right)+\sum_{i>j} C\left(Y_{i}, Y_{j}\right)$. Hence,

$$
\underset{n \rightarrow \infty}{\operatorname{LimSup}} \Pi\left(C_{D}(X ; n)\right) \leq \sum_{i=1}^{3} \operatorname{LimSup}_{n \rightarrow \infty}\left(\frac{U\left(Y_{i}\right)}{\log (n)}\right)+\sum_{i>j} \operatorname{LimSup}_{n \rightarrow \infty}\left(\frac{C\left(Y_{i}, Y_{j}\right)}{\log (n)}\right) .
$$

The remainder of the proof will consist of an examination of the terms on the right side of (1). 
We begin with $\operatorname{LimSup}_{n \rightarrow \infty}\left(\frac{U\left(Y_{2}\right)}{\log (n)}\right)$. Letting the point masses of $Y_{2}$ have coordinates $F^{-1}\left(\frac{L_{1}^{\tilde{n}}}{n}\right), F^{-1}\left(\frac{L_{1}^{\sim}+1}{n}\right), \ldots, F^{-1}\left(\frac{L_{2}^{\tilde{2}}}{n}\right)$ gives

$$
U\left(Y_{2}\right)=\frac{1}{n} \sum_{i=L_{1}+1}^{L_{2}} \sum_{j=L_{1}}^{i-1} \frac{1}{n\left(F^{-1}(i \sim / n)-F^{-1}\left(j^{\sim} / n\right)\right)} .
$$

Then, with $f=F^{\prime}$, Lemma 9.1, applied to the interval $\left[F^{-1}(\varepsilon), F^{-1}(1-\varepsilon)\right]$ yields

$$
U\left(Y_{2}\right)=\frac{1}{n} \sum_{i=L_{1}+1}^{L_{2}} \sum_{j=L_{1}}^{i-1} \frac{f\left(F^{-1}\left(i^{\sim} / n\right)\right)}{i-j}+\frac{1}{n^{2}} \sum_{i=L_{1}+1}^{L_{2}} \sum_{j=L_{1}}^{i-1} b\left(i^{\sim} / n, j^{\sim} / n\right) .
$$

The first double sum is bounded above by

$$
\frac{1}{n} \sum_{i=1}^{n} f\left(F^{-1}\left(\frac{i^{\sim}}{n}\right)\right) \sum_{j=1}^{n} \frac{1}{j} \leq \frac{\log (n)+1}{n} \sum_{i=1}^{n} f\left(F^{-1}\left(\frac{i^{\sim}}{n}\right)\right),
$$

where $\frac{1}{n} \sum_{i=1}^{n} f\left(F^{-1}\left(i^{\sim} / n\right)\right)$ is a Riemann sum for $\int_{0}^{1} f\left(F^{-1}(y)\right) d y=\int_{c}^{d} f^{2}(x) d x=$ $\Pi(X)$. Since $b\left(i^{\sim} / n, j^{\sim} / n\right)$ is bounded independent of $i^{\sim} / n$ and $j^{\sim} / n$

$$
\operatorname{Lim}_{n \rightarrow \infty}\left(\frac{1}{\log (n)}\left(\frac{1}{n^{2}} \sum_{i=L_{1}+1}^{L_{2}} \sum_{j=L_{1}}^{i-1} b\left(i^{\sim} / n, j^{\sim} / n\right)\right)\right)=0 .
$$

It follows that $\operatorname{LimSup}_{n \rightarrow \infty}\left(\frac{U\left(Y_{2}\right)}{\log (n)}\right) \leq \Pi(X)$.

In examining the remaining terms on the right side of (1), we will make use of the inequality $\frac{1}{F^{-1}(i \sim / n)-F^{-1}\left(j^{\sim} / n\right)}=\frac{f\left(F^{-1}(\xi)\right)}{(i / n-j / n)} \leq \frac{M}{(i / n-j / n)}$, where $\xi$ is between $i^{\sim / n}$ and $j^{\sim / n}$, and $M$ is an upper bound on $f(x)$ on $[c, d]$.

This leads to $U\left(Y_{1}\right) \leq \frac{M}{n} \sum_{i=2}^{L_{1}-1} \sum_{j=1}^{i-1} 1 /(i-j) \leq \frac{M}{n} \sum_{i=2}^{L_{1}-1}(\log (n)+1)$, and $U\left(Y_{3}\right) \leq \frac{M}{n} \sum_{i=L_{2}+2}^{n} \sum_{j=L_{2}+1}^{i-1} 1 /(i-j) \leq \frac{M}{n} \sum_{i=L_{2}+2}^{n}(\log (n)+1)$. Since $F^{-1}\left(\frac{\left.L_{1}^{\sim}-1\right)}{n}\right) \leq F^{-1}(\varepsilon)$ and $F^{-1}(1-\varepsilon) \leq F^{-1}\left(\frac{L_{2}^{\tilde{2}+1}}{n}\right)$, it follows that $L_{1} \leq n \varepsilon+1.5$ and $L_{2} \geq(1-\varepsilon) n-.5$. As a result, $\frac{U\left(Y_{1}\right)}{\log (n)} \leq \frac{M}{n}\left(\frac{\log (n)+1}{\log (n)}\right)(n \varepsilon-.5)$ and $\frac{U\left(Y_{3}\right)}{\log (n)} \leq$ $\frac{M}{n}\left(\frac{\log (n)+1}{\log (n)}\right)(n \varepsilon+.5)$. Hence, $\operatorname{LimSup}_{n \rightarrow \infty}\left(\frac{U\left(Y_{1}\right)}{\log (n)}\right) \leq M \varepsilon$ and $\operatorname{LimSup}_{n \rightarrow \infty}\left(\frac{U\left(Y_{3}\right)}{\log (n)}\right)$ $\leq M \varepsilon$.

An upper bound on $C\left(Y_{1}, Y_{2}\right)$ is obtained by placing all the mass of $Y_{1}$ at $F^{-1}\left(\frac{L_{1}^{\sim}-1}{n}\right)$ and then computing the cross potential of this point-mass and $Y_{2}$. Thus,

$$
\begin{aligned}
C\left(Y_{1}, Y_{2}\right) & \leq\left(\frac{L_{1}-1}{n}\right) M \sum_{j=L_{1}}^{L_{2}} \frac{1}{j-\left(L_{1}-1\right)} \\
& \leq\left(\frac{L_{1}-1}{n}\right) M(\log (n)+1) \leq\left(\varepsilon+\frac{.5}{n}\right) M(\log (n)+1) .
\end{aligned}
$$

Similarly,

$$
\begin{aligned}
C\left(Y_{3}, Y_{2}\right) & \leq\left(\frac{n-L_{2}}{n}\right) M \sum_{j=L_{1}}^{L_{2}} \frac{1}{L_{2}+1-j} \\
& \leq\left(\frac{n-L_{2}}{n}\right) M(\log (n)+1) \leq\left(\varepsilon+\frac{.5}{n}\right) M(\log (n)+1) .
\end{aligned}
$$


Placing all of the mass of $Y_{1}$ at $F^{-1}\left(\frac{L_{1}^{\sim}-1}{n}\right)$ and all of the mass of $Y_{3}$ at $F^{-1}\left(\frac{L_{2}^{\tilde{2}+1}}{n}\right)$ yields

$$
C\left(Y_{1}, Y_{3}\right) \leq\left(\frac{L_{1}-1}{n}\right)\left(\frac{n-L_{2}}{n}\right)\left(\frac{M}{\left(L_{2}+1\right) / n-\left(L_{1}-1\right) / n}\right) \leq\left(\varepsilon+\frac{.5}{n}\right)^{2} \frac{M}{(1-2 \varepsilon)}
$$

We conclude that $\operatorname{LimSup}_{n \rightarrow \infty} \frac{C\left(Y_{1}, Y_{2}\right)}{\log (n)} \leq M \varepsilon, \operatorname{LimSup}_{n \rightarrow \infty} \frac{C\left(Y_{3}, Y_{2}\right)}{\log (n)} \leq M \varepsilon$, and $\operatorname{LimSup}_{n \rightarrow \infty} \frac{C\left(Y_{1}, Y_{3}\right)}{\log (n)}=0$, and hence $\overline{\operatorname{Lim}} \Pi\left(C_{D}(X ; n)\right) \leq \Pi(X)+4 M \varepsilon$, thus establishing the result.

\section{THE TRUNCATION TRANSFORMATION FOR DISCRETE CONFIGURATIONS}

This transformation is the discrete analog of that appearing in Section 4.

Definition 10.1. Let $X=X_{A}$ be a standardized discrete configuration; then $r_{i}(X), i=0,1,2$, is defined as:

$$
r_{i}(X)=\sum_{\left|x_{j}\right|>\sqrt{5}} a_{j} x_{j}^{i}
$$

The truncation transformation is denoted $T(X)$ and defined for standardized discrete configurations as follows: if the point masses of $X$ that are in the interval $[-\sqrt{5}, \sqrt{5}]$ have in order the coordinates $x_{L}, x_{L+1}, \ldots, x_{R-1}, x_{R}$, then $T(X)$, contained in $[-\sqrt{5}, \sqrt{5}]$ is the $R-L+1$ point configuration defined by

$$
\begin{aligned}
T(X)=\left\{x_{L}, x_{L+1}, \ldots,\right. & x_{R-1}, x_{R} ; \\
& \left.\frac{a_{L}}{1-r_{0}(X)}, \frac{a_{L+1}}{1-r_{0}(X)}, \ldots, \frac{a_{R-1}}{1-r_{0}(X)}, \frac{a_{R}}{1-r_{0}(X)}\right\} .
\end{aligned}
$$

We note that $T(X)$ is well defined because Proposition 4.1 yields $1-r_{0}(X) \geq .8$.

$T$ will be applied to the members of a sequence of standardized collinear central configurations with dispersion less than or equal to $K$. The number of points in the configurations will tend to infinity and the normalized potentials will tend to the lower asymptotic normalized potential $\underline{\Pi}^{*}[K]$. In this section, we will show that when so applied, $T$ asymptotically maintains standardization, mass distribution, dispersion, and normalized potential, together with the added benefit that the transformed configurations are uniformly bounded.

Lemma 10.1. Let $X=X_{A}$ be a standardized $n$-point configuration with dispersion less than or equal to $K$, and let $l$ denote the number of points in $T(X)$.

Then

(i) The dispersion of $T(X)$ is less than or equal to $K$.

(ii) $l \geq \frac{n\left(1-r\left(X_{0}\right)\right)}{K} \geq \frac{.8 n}{K}$.

Proof. (i) This follows immediately from the definition of $T(X)$.

(ii) From Lemma 7.1, $a_{i} \leq \frac{K}{n}$. Using the notation of Definition 10.1, $1=$ $\sum_{j=L}^{R} \frac{a_{j}}{1-r_{0}(X)} \leq l \frac{K / n}{1-r_{0}(X)}$, and the result follows.

We now establish that $\underline{\Pi}^{*}[K]$ is positive.

Lemma 10.2. $\underline{\Pi}^{*}[K] \geq \frac{.32}{\sqrt{5} K^{4}}$. 
Proof. Let $X_{i}^{*}$ denote the standardized collinear central configuration $X_{A_{i}}^{*}$, where $A_{i}$ is an $n_{i}$ point mass vector and $d\left(A_{i}\right) \leq K$. Assume $n_{i} \rightarrow \infty$ and $\Pi\left(X_{i}^{*}\right) \rightarrow$ $\underline{\Pi}^{*}[K]$. Let $l_{i}$ denote the number of points in $T\left(X_{i}^{*}\right)$ and $r_{i}=r_{0}\left(X_{i}^{*}\right)$. Lemma 10.1 implies that $l_{i} \rightarrow \infty$.

By Lemma 7.1, the smallest possible mass in $X_{i}^{*}$ is $\frac{1}{K n_{i}}$, and hence each mass in $T\left(X_{i}^{*}\right)$ is at least $\frac{1}{K n_{i}\left(1-r_{i}\right)}$. Let $Y_{i}$ denote the $l_{i}$ point equal-mass configuration obtained by replacing each point-mass in $T\left(X_{i}^{*}\right)$ by a point-mass equal to $\frac{1}{K n_{i}\left(1-r_{i}\right)}$. (Note that the total mass of $Y_{i}$ is $\left.\frac{l_{i}}{K n_{i}\left(1-r_{i}\right)}\right)$. Given $\varepsilon>0$, it follows from Proposition 8.1 that for large $i, \Pi\left(Y_{i}\right) \geq \frac{(1-\varepsilon)}{2 \sqrt{5}}\left[\frac{l_{i}}{K n_{i}\left(1-r_{i}\right)}\right]^{2}$. As a result, since $\Pi\left(T\left(X_{i}^{*}\right)\right) \geq \Pi\left(Y_{i}\right)$, we have for large $i \Pi\left(X_{i}^{*}\right)=\frac{U\left(X_{i}^{*}\right)}{\log \left(n_{i}\right)} \geq \frac{\left(1-r_{i}\right)^{2} U\left(T\left(X_{i}^{*}\right)\right)}{\log \left(n_{i}\right)}=$ $\left[\frac{\log \left(l_{i}\right)\left(1-r_{i}\right)^{2}}{\log \left(n_{i}\right)}\right] \Pi\left(T\left(X_{i}^{*}\right)\right) \geq \frac{(1-\varepsilon)}{2 \sqrt{5}}\left(\frac{l_{i}}{n_{i}}\right)^{2}\left[\frac{\log \left(l_{i}\right)}{\log \left(n_{i}\right)}\right] \frac{1}{K^{2}} \geq\left(\frac{.32}{K^{4} \sqrt{5}}\right)(1-\varepsilon)\left(1+\frac{\log (.8 / K)}{\log \left(n_{i}\right)}\right)$, where we have used $\frac{l_{i}}{n_{i}} \geq \frac{8}{K}$. Since $\log \left(n_{i}\right) \rightarrow \infty, \underline{\Pi}^{*}[K]=\operatorname{Lim}_{i \rightarrow \infty} \Pi\left(X_{i}^{*}\right) \geq$ $\frac{.32}{K^{4} \sqrt{5}}$.

The next proposition is the analogue of Proposition 4.2. The function $W$ is defined in Definition 4.2 .

Proposition 10.1. If $X=X_{A}$ is an n-point standardized configuration for which $d(A) \leq K$ and $n>1.25 K$, then

$$
\Pi\left(T^{S}(X)\right) \leq \frac{\sqrt{W\left(r_{0}(X)\right)} \Pi(X)}{1-\log (1.25 K) / \log (n)} .
$$

Proof. On setting $r_{i}=r_{i}(X)$, as in the proof of Proposition 4.2, we find $\sigma^{2}(T(X))=$ $\frac{1-r_{2}}{1-r_{0}}-\frac{\left(0-r_{1}\right)^{2}}{\left(1-r_{0}\right)^{2}}$. Let $l$ denote the number of points in $T(X)$.

Using $\Pi(T(X)) \leq \frac{\log (n)}{\log (l)}\left[\frac{\Pi(X)}{\left(1-r_{0}\right)^{2}}\right]$ and $\frac{\log (n)}{\log (l)} \leq \frac{1}{1-\log (1.25 K) / \log (n)}$, which follows from Lemma 10.1, we have

$$
\begin{aligned}
\Pi\left(T^{S}(X)\right) & =\sigma(T(X)) \Pi(T(X)) \\
& \leq \sqrt{\frac{1-r_{2}}{1-r_{0}}-\frac{\left(0-r_{1}\right)^{2}}{\left(1-r_{0}\right)^{2}}}\left(\frac{1}{1-\log (1.25 K) / \log (n)}\right) \frac{\Pi(X)}{\left(1-r_{0}\right)^{2}} .
\end{aligned}
$$

Thus, $\Pi\left(T^{*} S(X)\right) \leq \sqrt{\frac{1-r_{2}}{1-r_{0}}}\left(\frac{1}{1-\log (1.25 K) / \log (n)}\right) \frac{\Pi(X)}{\left(1-r_{0}\right)^{2}}$. The result follows on noting that, as in the proof of Proposition 4.2, $r_{2} \geq 5 r_{0}$.

Proposition 10.2. Let $\left\{X_{i}\right\}_{i=1}^{\infty}$ be a sequence of standardized $n_{i}$-point configurations for which $n_{i} \rightarrow \infty, \operatorname{Lim}_{i \rightarrow \infty} \Pi\left(X_{i}\right)=\underline{\Pi}^{*}[K]$, and each configuration has dispersion less than or equal to $K$. Then, if $Z_{i}=T\left(X_{i}\right)$ and $F_{i}$ and $G_{i}$ are respectively the mass distribution functions for $X_{i}$ and $Z_{i}$,

(i) $\operatorname{Lim}_{i \rightarrow \infty} \mu\left(Z_{i}\right)=0$.

(ii) $\operatorname{Lim}_{i \rightarrow \infty} \sigma\left(Z_{i}\right)=1$.

(iii) $\operatorname{Lim}_{i \rightarrow \infty} \Pi\left(Z_{i}\right)=\underline{\Pi}^{*}[K]$.

(iv) $\operatorname{Lim}_{i \rightarrow \infty}\left\|F_{i}-G_{i}\right\|_{S}=0$.

Proof. (i), (ii) We note that since the dispersion of $Z_{i}^{S}$ is less than or equal to $K$, it is impossible for $\operatorname{Lim} \Pi\left(Z_{i}^{S}\right)$ to be less than $\underline{\Pi}^{*}[K]$. But if $\operatorname{Lim}_{i \rightarrow \infty} r_{0}\left(X_{i}\right) \neq 0$, then by Proposition 10.1 in conjunction with Lemmas 10.2 and 4.1 , this would be the case. Hence, $\operatorname{Lim}_{i \rightarrow \infty} r_{0}\left(X_{i}\right)=0$. 
On using $r_{j i}=r_{j}\left(X_{i}\right)$, for $n_{i}>1.25 K$ the proof of Proposition 10.1 gives

$$
\Pi\left(Z_{i}^{s}\right) \leq \sqrt{\frac{1-r_{2 i}}{1-r_{0 i}}-\frac{\left(0-r_{1 i}\right)^{2}}{\left(1-r_{0 i}\right)^{2}}}\left(\frac{1}{1-\log (1.25 K) / \log \left(n_{i}\right)}\right) \frac{\Pi\left(X_{i}\right)}{\left(1-r_{0 i}\right)^{2}} .
$$

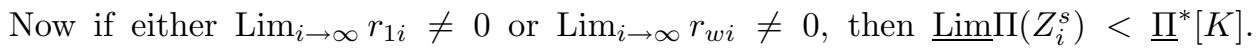
Hence, $\operatorname{Lim}_{i \rightarrow \infty} r_{1 i}=\operatorname{Lim}_{i \rightarrow \infty} r_{2 i}=0$, and from $\mu\left(Z_{i}\right)=\frac{-r_{1 i}}{1-r_{0 i}}$ and $\sigma\left(Z_{i}\right)=$ $\sqrt{\frac{1-r_{2 i}}{1-r_{0 i}}-\frac{\left(0-r_{1 i}\right)^{2}}{\left(1-r_{0 i}\right)^{2}}}$, we conclude that $\operatorname{Lim}_{i \rightarrow \infty} \mu\left(Z_{i}\right)=0$ and $\operatorname{Lim}_{i \rightarrow \infty} \sigma\left(Z_{i}\right)=1$.

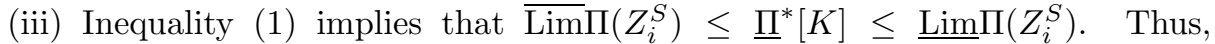
$\operatorname{Lim}_{i \rightarrow \infty} \Pi\left(Z_{i}\right)=\operatorname{Lim}_{i \rightarrow \infty} \frac{1}{\sigma\left(Z_{i}\right)} \operatorname{Lim}_{i \rightarrow \infty} \Pi\left(Z_{i}^{S}\right)=\underline{\Pi}^{*}[K]$.

(iv) Let $X=X_{A}$ be any standardized $n$-point configuration, and let $F(x)$ and $G(x)$ respectively represent the mass distribution functions for $X$ and $T(X)$. Set $r_{0}=r_{0}(X)$, and let $x_{L}, x_{L+1}, \ldots, x_{R}$ be the coordinates of the point-masses of $X$ that are in $[-\sqrt{5}, \sqrt{5}]$.

If $x<x_{L}$, then $G(x)=0$ and $F(x) \leq r_{0}$. If $x \geq x_{R}$, then $G(x)=1$ and $F(x) \geq 1-r_{0}$. If $x_{j} \leq x<x_{j+1}, j=L, \ldots, R-1, F(x)=\sum_{k=1}^{j} a_{k}$, and $G(x)=\sum_{k=L}^{j} \frac{a_{k}}{1-r_{0}}$. So, $|F(x)-G(x)| \leq \sum_{k=1}^{L-1} a_{k}+\left(\frac{1}{1-r_{0}}-1\right) \sum_{k=L}^{j} a_{k} \leq r_{0}+\frac{r_{0}}{1-r_{0}}$. Thus, in any case, $|F(x)-G(x)| \leq \frac{2 r_{0}}{1-r_{0}}$, and so $\left\|F_{i}-G_{i}\right\|_{S} \leq \frac{2 r_{0 i}}{1-r_{0 i}}$. Since $r_{0 i} \rightarrow 0$, the result follows.

\section{Uniform CONVERgence: The Proof}

Our goal is to show that $F^{*}$, the mass distribution function for $X^{*}$, is the uniform limit of the mass distribution functions of standardized collinear central configurations of bounded dispersion. We begin by determining the formula for $F^{*}$, and then, following the approach outlined in Section 6, establish versions of key results (i) and (ii) in Theorems 11.2 and 11.3.

Theorem 11.1. The mass distribution function, $F^{*}$, for the standardized continuous collinear central configuration $X^{*}$ is given by

$$
F^{*}(x)=\left\{\begin{array}{l}
\frac{1}{2}+\frac{\sqrt{5}}{100}\left(15 x-x^{3}\right):-\sqrt{5} \leq x \leq \sqrt{5} \\
1: x>\sqrt{5} \\
0: x<-\sqrt{5}
\end{array}\right.
$$

Proof. This follows from integrating the density function $f^{*}(x)$ given in Theorem 2.1.

Lemma 11.1. If $\left\{Z_{i}\right\}_{i=1}^{\infty}$ is a sequence of either discrete or continuous configurations, each consisting of more than a single point and for which $\operatorname{Lim}_{i \rightarrow \infty} \sigma\left(Z_{i}\right)=1$ and $\left\{\Pi\left(Z_{i}\right)\right\}_{i=1}^{\infty}$ are bounded, and if $X_{i}=Z_{i}^{S}$, then $\operatorname{LimSup}_{i \rightarrow \infty} \Pi\left(Z_{i}\right)=$ $\operatorname{LimSup}_{i \rightarrow \infty} \Pi\left(X_{i}\right)$.

Proof. From Proposition 2.3, $\Pi\left(Z_{i}^{S}\right)=\sigma\left(Z_{i}\right) \Pi\left(Z_{i}\right)$. Thus, $\left|\Pi\left(Z_{i}\right)-\Pi\left(Z_{i}^{S}\right)\right|=$ $\left|\sigma\left(Z_{i}\right)-1\right| \Pi\left(Z_{i}\right) \rightarrow 0$, and the result follows.

Lemma 11.2. If $K \geq 1, \frac{1}{K^{5 / 2}} \Pi\left(X_{n}^{*}\right) \leq \Pi^{*}[K ; n] \leq \bar{\Pi}^{*}[K ; n] \leq K^{5 / 2} \Pi\left(X_{n}^{*}\right)$, where $X_{n}^{*}$ denotes the $n$-point equal-mass standardized collinear central configuration. 
Proof. Let $A \in M_{n}[K]$ and $Y_{A}^{*}=\left\{y_{1}, y_{2}, \ldots, y_{n} ; a_{1}, a_{2}, \ldots, a_{n}\right\}$ be the corresponding $n$-point standardized collinear central configuration. Replacing $A$ by the equalmass vector $E$, let

$$
Y_{E}=\left\{y_{1}, y_{2}, \ldots, y_{n} ; \frac{1}{n}, \frac{1}{n}, \ldots, \frac{1}{n}\right\} .
$$

Set $X_{n}^{*}=\left\{x_{1}, x_{2}, \ldots, x_{n} ; \frac{1}{n}, \frac{1}{n}, \ldots, \frac{1}{n}\right\}$, and then replacing the equal-mass vector by $A$, let

$$
X_{A}=\left\{x_{1}, x_{2}, \ldots, x_{n} ; a_{1}, a_{2}, \ldots, a_{n}\right\} .
$$

From Lemma 7.1 we have $\frac{1}{K n} \leq a_{i} \leq \frac{K}{n}$. As a result, $\sigma^{2}\left(X_{A}\right) \leq \sum_{i=1}^{n} a_{i} x_{i}^{2} \leq$ $K \sum_{i=1}^{n} \frac{1}{n} x_{i}^{2}=K$, and $\sigma^{2}\left(Y_{E}\right) \leq \sum_{i=1}^{n} \frac{1}{n} y_{i}^{2} \leq K \sum_{i=1}^{n} a_{i} y_{i}^{2}=K$. We then have $\Pi\left(Y_{A}^{*}\right) \geq \frac{1}{K^{2}} \Pi\left(Y_{E}\right)=\frac{1}{K^{2} \sigma\left(Y_{E}\right)} \Pi\left(Y_{E}^{S}\right) \geq \frac{1}{K^{2} \sigma\left(Y_{E}\right)} \Pi\left(X_{n}^{*}\right) \geq \frac{1}{K^{5 / 2}} \Pi\left(X_{n}^{*}\right)$ and $\Pi\left(Y_{A}^{*}\right) \leq$ $\Pi\left(X_{A}^{S}\right)=\sigma\left(X_{A}\right) \Pi\left(X_{A}\right) \leq \sigma\left(X_{A}\right) K^{2} \Pi\left(X_{n}^{*}\right) \leq K^{5 / 2} \Pi\left(X_{n}^{*}\right)$, thus establishing the result.

Theorem 11.2 (Uniform Convergence of Normalized Potentials). For fixed $\varepsilon>0$ and $K \geq 1$, there exists an integer $N$, such that if any given standardized collinear central configuration $Y^{*}$ of dispersion less than or equal to $K$ has more than $N$ points, then its normalized potential $\Pi\left(Y^{*}\right)$ satisfies $\left|\Pi\left(Y^{*}\right)-\frac{3 \sqrt{5}}{25}\right|<\varepsilon$. Equivalently, if $K \geq 1, \bar{\Pi}^{*}[K]=\underline{\Pi}^{*}[K]=\Pi^{*}=\frac{3 \sqrt{5}}{25}$.

Proof. From Theorem 2.1 and Proposition 3.4, $\Pi^{*}=\Pi_{\sqrt{5}}^{*}=\frac{3 \sqrt{5}}{25}$. The proof of the remainder of the second of the equivalent formulations will be divided into two parts.

Part $A . \Pi^{*} \geq \bar{\Pi}^{*}[K]$ :

From Lemma $11.2, \bar{\Pi}^{*}[K ; n]$ is finite. Noting a priori that

$$
\bar{\Pi}^{*}[K]=\operatorname{LimSup}_{n \rightarrow \infty} \bar{\Pi}^{*}[K ; n]
$$

might possibly be $+\infty$, let $\left\{W_{i}^{*}\right\}_{i=1}^{\infty}$ be a given sequence of $l_{i}$-point standardized collinear central configurations of dispersion less than or equal to $K$ with mass vectors $\left\{B_{i}\right\}_{i=1}^{\infty}$, for which $l_{i} \rightarrow \infty, l_{i}>e^{K}$, and $\Pi\left(W_{i}^{*}\right) \rightarrow \bar{\Pi}^{*}[K]$. Set $Z_{0 i}=$ $C_{D}\left(X^{*} ;\left[l_{i} \log \left(l_{i}\right)\right]+1\right), X_{0 i}=Z_{0 i}^{S}, Z_{1 i}=K_{F}\left(X_{0 i}: B_{i}\right)$, and $X_{1 i}=Z_{1 i}^{S}$. We note that the standardizations $X_{0 i}$ and $X_{1 i}$ exist because $Z_{0 i}$ and $Z_{1 i}$ each contain more than a single point. Hence, $X_{0 i}$ is an $\left[l_{i} \log \left(l_{i}\right)\right]+1$-point standardized equal-mass configuration and $X_{1 i}$ is an $l_{i}$-point standardized configuration with mass vector $B_{i}$.

Using Lemma 11.1 and results related to the configuration transformations, we can establish the inequalities in (1).

(1) $\Pi^{*} \geq \operatorname{LimSup}_{i \rightarrow \infty} \Pi\left(Z_{0 i}\right)=\underset{i \rightarrow \infty}{\operatorname{LimSup}} \Pi\left(X_{0 i}\right) \geq \operatorname{LimSup}_{i \rightarrow \infty} \Pi\left(Z_{1 i}\right)=\operatorname{LimSup}_{i \rightarrow \infty} \Pi\left(X_{1 i}\right)$.

The justification is as follows: Since $X^{*}$ and $F^{*}$ on $[-\sqrt{5}, \sqrt{5}]$ satisfy the hypotheses of Proposition 9.1, the first inequality is an immediate consequence of part (ii) of this proposition. The next equality follows from Lemma 11.1, because by Proposition 9.1, $\operatorname{Lim}_{i \rightarrow \infty} \sigma\left(Z_{0 i}\right)=1$ and $\left\{\Pi\left(Z_{0 i}\right)\right\}_{i=1}^{\infty}$ is bounded. To establish the second inequality, we begin by noting that Proposition 9.1 also yields $\operatorname{Lim}_{i \rightarrow \infty} \mu\left(Z_{0 i}\right)=0$. As a result, since $Z_{0 i}$ is contained in $[-\sqrt{5}, \sqrt{5}]$, we conclude that there is a finite interval $[-c, c]$ containing each of the configurations in the sequence $\left\{X_{0 i}\right\}_{i=1}^{\infty}$. Thus 
$\left\{B_{i}\right\}_{i=1}^{\infty}$ and $\left\{X_{0 i}\right\}_{i-1}^{\infty}$ satisfy all of the hypotheses of Proposition 7.6, and so part (i) of the proposition gives the second inequality. The final inequality in (1) follows from Lemma 11.1 because $\left\{\Pi\left(Z_{1 i}\right)\right\}_{i=1}^{\infty}$ is bounded and Proposition 7.6, part (iii) gives $\operatorname{Lim}_{i \rightarrow \infty} \sigma\left(Z_{1 i}\right)=1$.

The proof of Part $\mathrm{A}$ is completed on noting that $X_{1 i}$ is a standardized $l_{i}$-point configuration with mass vector $B_{i}$. Its normalized potential is thus greater than or equal to that of $W_{i}^{*}$. Hence, $\operatorname{LimSup}_{i \rightarrow \infty} \Pi\left(X_{1 i}\right) \geq \operatorname{Lim}_{i \rightarrow \infty} \Pi\left(W_{i}^{*}\right)=\bar{\Pi}^{*}[K]$, and hence from (1), $\Pi^{*} \geq \bar{\Pi}^{*}[K]$.

Part B. $\underline{\Pi}^{*}[K] \geq \Pi^{*}$ :

Let $\left\{X_{i}^{*}\right\}_{i=1}^{\infty}$ be a given sequence of $n_{i}$-point standardized collinear central configurations of dispersion less than or equal to $K$ for which $n_{i} \rightarrow \infty, n_{i}>K$. $\operatorname{Max}\left[810,1.25 e^{K}\right]$, and $\Pi\left(X_{i}^{*}\right) \rightarrow \underline{\Pi}^{*}[K]=\operatorname{LimInf}_{n \rightarrow \infty} \underline{\Pi}^{*}[K ; n]$. Using the transformation sequence of Procedure 6.1, set $X_{0 i}=X_{i}^{*}, Z_{1 i}=T\left(X_{0 i}\right), X_{1 i}=Z_{1 i}^{S}$, $Z_{2 i}=K_{T}\left(X_{1 i}\right), X_{2 i}=Z_{2 i}^{S}, Z_{3 i}=H\left(X_{2 i}\right)$, and $X_{3 i}=Z_{3 i}^{S}$.

We first examine the number of points in the discrete configurations to show that they tend to infinity and to verify that the $X_{j i}$ and $Z_{j i}$ are well defined. For $j=1,2$, the number of points in $X_{j i}$ and $Z_{j i}$ are equal. Let $l_{j i}$ denote this common number. From part (ii) of Lemma 10.1, $l_{1 i} \geq \frac{.8 n_{i}}{K}>\operatorname{Max}\left[648, e^{K}\right]$. Thus $l_{1 i} \rightarrow \infty$, and further, it follows form part (i) of Proposition 7.4 that $Z_{2 i}$ is well defined, and from Definition 7.2 that $l_{2 i}=\left[\frac{l_{1 i}}{\log \left(l_{1 i}\right)}\right]$. Thus, $l_{2 i} \rightarrow \infty$, and $l_{2 i}>\frac{648}{\log (648)}-1 \approx 99.0944$. Hence, $l_{2 i} \geq 100$, and so, according to Section $8, Z_{3 i}$ is well defined, as is $X_{3 i}$.

We can now establish the equalities and inequalities in (2), (3), and (4).

$$
\underline{\Pi}^{*}[K]=\operatorname{Lim}_{i \rightarrow \infty} \Pi\left(X_{0 i}\right)=\operatorname{Lim}_{i \rightarrow \infty} \Pi\left(Z_{1 i}\right)=\operatorname{Lim}_{i \rightarrow \infty} \Pi\left(X_{1 i}\right) .
$$

In (2), the first is by definition and the second by part (iii) of Proposition 10.2, since $\left\{X_{0 i}\right\}_{i=1}^{\infty}$ satisfies all the hypotheses of that proposition. The final equality of (2) follows from Proposition 2.3, because by part (ii) of Proposition 10.2, $\operatorname{Lim}_{i \rightarrow \infty} \sigma\left(Z_{1 i}\right)=1$.

$$
\operatorname{Lim}_{i \rightarrow \infty} \Pi\left(X_{1 i}\right) \geq \operatorname{LimSup}_{i \rightarrow \infty} \Pi\left(Z_{2 i}\right)=\operatorname{LimSup}_{i \rightarrow \infty} \Pi\left(X_{2 i}\right) \geq \operatorname{LimSup}_{i \rightarrow \infty} \Pi\left(Z_{3 i}\right) .
$$

To establish the first inequality in (3), we begin by noting that Proposition 10.2 also yields $\operatorname{Lim}_{i \rightarrow \infty} \mu\left(Z_{1 i}\right)=0$. As a result, since $Z_{1 i}$ is contained in $[-\sqrt{5}, \sqrt{5}]$, we conclude that there is a finite interval $[-c, c]$ containing each of the configurations in the sequence $\left\{X_{1 i}\right\}_{i=1}^{\infty}$. Further, by part (i) of Lemma 10.1, the $Z_{1 i}$, and hence the $X_{1 i}$, have dispersion less than or equal to $K$, and thus the sequence $\left\{X_{1 i}\right\}_{i=1}^{\infty}$ satisfies all of the hypotheses of Proposition 7.5. So part (i) of this proposition gives the inequality. The first equality in (3) follows from Lemma 11.1 because $\left\{\Pi\left(Z_{2 i}\right)\right\}_{i=1}^{\infty}$ is bounded and Proposition 7.5 gives $\operatorname{Lim}_{i \rightarrow \infty} \sigma\left(Z_{2 i}\right)=1$ as well as $\operatorname{Lim}_{i \rightarrow \infty} \mu\left(Z_{2 i}\right)=0$. To prove the final inequality in (3), we first note that parts (ii), (iii), and (v) of Proposition 7.5 imply that there is a finite interval $\left[-c_{1}, c_{1}\right]$ which contains each member of the sequence $\left\{X_{2 i}\right\}_{i=1}^{\infty}$. Thus the sequence satisfies all the hypotheses of Proposition 8.2, and so part (i) yields the inequality.

$$
\operatorname{LimSup}_{i \rightarrow \infty} \Pi\left(Z_{3 i}\right)=\underset{i \rightarrow \infty}{\operatorname{LimSup}} \Pi\left(X_{3 i}\right) \geq \Pi^{*} .
$$

In (4), the first equality follows from Lemma 11.1, because by part (iii) of Proposition 8.2, $\operatorname{Lim}_{i \rightarrow \infty} \sigma\left(Z_{3 i}\right)=1$. The final inequality is an immediate consequence of the fact that for all $i, X_{3 i} \in S_{\infty}$, and hence $\Pi\left(X_{3 i}\right) \geq \Pi^{*}$. 
Combining (2), (3), and (4), yields the result in Part B, and thus completes the proof of the theorem, since $\bar{\Pi}^{*}[K] \geq \underline{\Pi}^{*}[K]$.

Theorem 11.3. If $c \geq \sqrt{5}$ and $X$ is a standardized continuous configuration contained in $[-c, c]$ with mass distribution function $F$, then

$$
\left\|F-F^{*}\right\|_{S} \leq \sqrt{2 c} \sqrt{\Pi(X)-\Pi^{*}} .
$$

Proof. Let $f \in D(R)$ denote the mass density function for $X$. We will begin by showing that $\left\|f-f^{*}\right\|_{2}^{2} \leq \Pi(X)-\Pi^{*}$.

Let $\delta$ be defined by $\left\|f-f^{*}\right\|_{2}^{2}=\Pi(X)-\Pi^{*}+\delta, \varepsilon>0$ small, and define $g=(1-\varepsilon) f^{*}+\varepsilon f$. Since $D(R)$ is convex, $g \in D(R)$. We have

$$
\|g\|_{2}^{2}=\left\|f^{*}-\varepsilon\left(f^{*}-f\right)\right\|_{2}^{2}=\left\|f^{*}\right\|_{2}^{2}-2 \varepsilon\left\langle f^{*}, f^{*}-f\right\rangle+\varepsilon^{2}\left\|f^{*}-f\right\|_{2}^{2} .
$$

On using the identity $2\left\langle f^{*}-f, f^{*}\right\rangle=\left\|f-f^{*}\right\|_{2}^{2}-\left(\Pi(X)-\Pi^{*}\right)$, we get $\|g\|_{2}^{2}=\Pi^{*}-$ $\varepsilon\left(\delta-\varepsilon\left\|f-f^{*}\right\|_{2}^{2}\right)$. If $\delta>0, \varepsilon$ could be chosen small enough so that $\|g\|_{2}^{2}<\Pi^{*}$. But, since this inequality is not possible, it follows that $\delta \leq 0$ and $\left\|f-f^{*}\right\|_{2}^{2} \leq \Pi(X)-\Pi^{*}$.

If $x \notin[-c, c],\left|F(x)-F^{*}(x)\right|=0$. For $x \in[-c, c]$ we have $\left|F(x)-F^{*}(x)\right|=$ $\left|\int_{-c}^{x}\left(f(t)-f^{*}(t)\right) d t\right| \leq \int_{-c}^{c}\left|f(t)-f^{*}(t)\right| d t$. Applying the Schwarz inequality to the last integral yields

$$
\begin{aligned}
\left|F(x)-F^{*}(x)\right| & \leq \sqrt{\int_{-c}^{c}\left|f(t)-f^{*}(t)\right|^{2} d t} \sqrt{\int_{-c}^{c} 1 d t} \\
& =\sqrt{2 c}\left\|f-f^{*}\right\|_{2} \leq \sqrt{2 c} \sqrt{\Pi(X)-\Pi^{*}} .
\end{aligned}
$$

Prior to completing the proof of uniform convergence in Theorem 11.4, we need a final lemma relating standardization and uniform convergence to $F^{*}$. This will enable us to transfer uniform convergence from a standardized configuration sequence to the unstandardized version.

Lemma 11.3. Let $\left\{Z_{i}\right\}_{i=1}^{\infty}$ be a sequence of configurations (either discrete or continuous). Set $X_{i}=Z_{i}^{S}$, and let $G_{i}$ denote the mass distribution function for $Z_{i}$, and $F_{i}$ the mass distribution function for $X_{i}$. Then if $\mu\left(Z_{i}\right) \rightarrow 0, \sigma\left(Z_{i}\right) \rightarrow 1$, and $\left\|F_{i}-F^{*}\right\|_{S} \rightarrow 0$, it follows that $\left\|G_{i}-F^{*}\right\|_{S} \rightarrow 0$.

Proof. Let $\varepsilon>0$ be given, and set $\mu_{i}=\mu\left(Z_{i}\right)$ and $\sigma_{i}=\sigma\left(Z_{i}\right)$.

Since $F^{*}$ is uniformly continuous on $[-7,7]$, there is a $\delta, 0<\delta<1$, such that $x, y \in[-7,7]$ and $|x-y|<\delta$ implies that $\left|F^{*}(x)-F^{*}(y)\right|<\frac{\varepsilon}{2}$. Choose $N$ so that $i>N$ implies

(i) $\left\|F^{*}-F_{i}\right\|_{S}<\frac{\varepsilon}{2}$ and

(ii) $6\left|\frac{1-\sigma_{i}}{\sigma_{i}}\right|+\frac{\left|\mu_{i}\right|}{\sigma_{i}}<\delta<1$.

Note that $i>N$ also implies $\left|\frac{1-\sigma_{i}}{\sigma_{i}}\right|<\frac{1}{4}$ and $\frac{\left|\mu_{i}\right|}{\sigma_{i}}<\frac{6}{4}$.

Since $G_{i}(x)=F_{i}\left(\frac{x-\mu_{i}}{\sigma_{i}}\right)$, we have $\left|G_{i}(x)-F^{*}(x)\right|=\left|F_{i}\left(\frac{x-\mu_{i}}{\sigma_{i}}\right)-F^{*}(x)\right| \leq$ $\left|F_{i}\left(\frac{x-\mu_{i}}{\sigma_{i}}\right)-F^{*}\left(\frac{x-\mu_{i}}{\sigma_{i}}\right)\right|+\left|F^{*}\left(\frac{x-\mu_{i}}{\sigma_{i}}\right)-F^{*}(x)\right| \leq\left\|F_{i}-F^{*}\right\|_{S}+\left|F^{*}\left(\frac{x-\mu_{i}}{\sigma_{i}}\right)-F^{*}(x)\right|$.

Now if $|x| \leq 6$ and $i>N$, then $\left|x-\frac{x-\mu_{i}}{\sigma_{i}}\right| \leq 6\left|\frac{1-\sigma_{i}}{\sigma_{i}}\right|+\frac{\left|\mu_{i}\right|}{\sigma_{i}}<\delta<1$, and this implies that $\left|F^{*}\left(\frac{x-\mu_{i}}{\sigma_{i}}\right)-F^{*}(x)\right|<\frac{\varepsilon}{2}$. While if $|x|>6$ and $i>N$, then $\left|x-\frac{x-\mu_{i}}{\sigma_{i}}\right| \leq$ $|x|\left|\frac{1-\sigma_{i}}{\sigma_{i}}\right|+\frac{\left|\mu_{i}\right|}{\sigma_{i}} \leq \frac{|x|}{4}+\frac{6}{4} \leq \frac{|x|}{2}$, and hence $x$ and $\frac{x-\mu_{i}}{\sigma_{i}}$ have the same sign and both exceed 3 in magnitude. It follows, in this case, that $\left|F^{*}\left(\frac{x-\mu_{i}}{\sigma_{i}}\right)-F^{*}(x)\right|=0$. So, in any case, if $i>N,\left|G_{i}(x)-F^{*}(x)\right| \leq\left\|F_{i}-F^{*}\right\|_{S}+\left|F^{*}\left(\frac{x-\mu_{i}}{\sigma_{i}}\right)-F^{*}(x)\right|<\varepsilon$. This completes the proof. 
With the necessary lemmas in place and Theorems 11.2 and 11.3 giving key results (i) and (ii) of Section 6, the proof of uniform convergence can now be implemented using the Proof Strategy from that section.

Theorem 11.4 (Uniform Convergence of Mass Distributions). For fixed $\varepsilon>0$ and $K \geq 1$, there exists an integer $N$, such that if any given standardized collinear central configuration of dispersion less than or equal to $K$ has more than $N$ points, then its mass distribution function $F(x)$ satisfies $\left\|F-F^{*}\right\|_{S}<\varepsilon$.

Proof. Let $K \geq 1$ be any fixed value, and let $\left\{X_{i}^{*}\right\}_{i=1}^{\infty}$ be any given sequence of $n_{i^{-}}$ point standardized collinear central configurations of dispersion less than or equal to $K$ for which $n_{i} \rightarrow \infty$ and $n_{i}>K \cdot \operatorname{Max}\left[810,1.25 e^{K}\right]$. The theorem will follow if we can show that $\left\|F_{i}-F^{*}\right\|_{S} \rightarrow 0$, where $F_{i}$ is the mass distribution function for $X_{i}^{*}$. For if it were false, there would exist some $K \geq 1$ and $\varepsilon>0$ and a sequence of collinear central configurations as above for which the corresponding mass distribution functions $\left\{F_{i}\right\}_{i-1}^{\infty}$ satisfy $\left\|F_{n}-F^{*}\right\|_{S}>\varepsilon$ for all $i$.

The method of proof that we will use to show that $\left\|F_{i}-F^{*}\right\|_{S} \rightarrow 0$ is essentially that of the Proof Strategy of Section 6 . To begin, we note that it follows immediately from Theorem 11.2 that for large $i, \Pi\left(X_{i}^{*}\right) \approx \Pi^{*}$, i.e. $\Pi\left(X_{i}^{*}\right) \rightarrow \Pi^{*}=\underline{\Pi}^{*}[K]$, and thus $\left\{X_{i}^{*}\right\}_{i=1}^{\infty}$ can be viewed as the sequence appearing in Part B of the proof of that theorem. As in that proof, and again using the transformation sequence of Procedure 6.1, set $X_{0 i}=X_{i}^{*}, Z_{1 i}=T\left(X_{0 i}\right), X_{1 i}=Z_{1 i}^{S}, Z_{2 i}=K_{T}\left(X_{1 i}\right), X_{2 i}=Z_{2 i}^{S}$, $Z_{3 i}=H\left(X_{2 i}\right)$, and $X_{3 i}=Z_{3 i}^{S}$, and let $F_{j i}, j=0,1,2,3$, denote the mass distribution function for $X_{j i}$, and $G_{j i}, j=1,2,3$, the mass distribution function for $Z_{j i}$. $X_{3 i}$ is the standardization histogram configuration constructed from $X_{i}^{*}$ after some alteration of $X_{i}^{*}$ via the transformations $T$ and $K_{T}$.

From (2), (3), and (4) in the proof of Theorem 11.2, we find that

$$
\operatorname{LimSup}_{i \rightarrow \infty} \Pi\left(X_{3 i}\right)=\Pi^{*},
$$

and since $\Pi\left(X_{3 i}\right) \geq \Pi^{*}$, it follows that $\operatorname{Lim}_{i \rightarrow \infty} \Pi\left(X_{3 i}\right)=\Pi^{*}$. In line with the Proof Strategy, we can also take the point of view that (2), (3), and (4) show that $\Pi\left(X_{3 i}\right) \approx \Pi\left(X_{i}^{*}\right)$ for large $i$.

To apply Theorem 11.3 to conclude that for large $i$ the mass distribution function for $X_{3 i}$ is uniformly close to $F^{*}$, we must first show that each member of the sequence $\left\{X_{3 i}\right\}_{i=1}^{\infty}$ is contained in a fixed finite interval. In the proof of Part B of Theorem 11.2, it is shown that each member of $\left\{X_{2 i}\right\}_{i=1}^{\infty}$ is contained in $\left[-c_{1}, c_{1}\right]$, and so on applying part (v) of Proposition 8.2, we find each member of $\left\{Z_{3 i}\right\}_{i-1}^{\infty}$ is contained in $\left[-c_{1}, c_{1}\right]$. Parts (ii) and (iii) of the same proposition give $\sigma\left(Z_{3 i}\right) \rightarrow 1$ and $\mu\left(Z_{3 i}\right) \rightarrow 0$, and hence we can conclude that there is an interval $\left[-c_{2}, c_{2}\right]$ which contains each member of $\left\{X_{3 i}\right\}_{i=1}^{\infty}$. Further, we can assume $c_{2}>\sqrt{5}$. Invoking Theorem 11.3 yields $\operatorname{Lim}_{i \rightarrow \infty}\left\|F_{3 i}-F^{*}\right\|_{S}=0$.

To complete the proof we must now verify that when $i$ is large, the mass distribution for $X_{3 i}$ is approximately that of $X_{i}^{*}$. We will accomplish this by working backwards through the configuration transformations. We will show that if the mass distribution functions of a transformed sequence of configurations have $F^{*}$ as a uniform limit, then so do the mass distribution functions of the original sequence.

To begin, we note from above and from the proof of Part B of Theorem 11.2 that $\operatorname{Lim}_{i \rightarrow \infty} \sigma\left(Z_{j i}\right)=1$ and $\operatorname{Lim}_{i \rightarrow \infty} \mu\left(Z_{j i}\right)=0, j=1,2,3$. Applying Lemma 11.3, we see that for $j=1,2$, or 3 , if $\left\|F_{j i}-F^{*}\right\|_{S} \rightarrow 0$, then $\left\|G_{j i}-F^{*}\right\|_{S} \rightarrow 0$. Hence, immediately we conclude that $\operatorname{Lim}_{i \rightarrow \infty}\left\|G_{3 i}-F^{*}\right\|_{S}=0$. Again using the proof 
of Part B of Theorem 11.2, we note that $\left\{X_{2 i}\right\}_{i=1}^{\infty}$ satisfies all the hypotheses of Proposition 8.2, $\left\{X_{1 i}\right\}_{i=1}^{\infty}$ all the hypotheses of Proposition 7.5, and $\left\{X_{0 i}\right\}_{i=1}^{\infty}$ all the hypotheses of Proposition 10.2. Invoking part (iv) of Proposition 8.2, we conclude that $\operatorname{Lim}_{i \rightarrow \infty}\left\|G_{3 i}-F^{*}\right\|_{S}=0$ implies $\operatorname{Lim}_{i \rightarrow \infty}\left\|F_{2 i}-F^{*}\right\|_{S}=0$. From the above, this implies that $\operatorname{Lim}_{i \rightarrow \infty}\left\|G_{2 i}-F^{*}\right\|_{S}=0$, which in turn, on invoking part (iv) of Proposition 7.5, implies that $\operatorname{Lim}_{i \rightarrow \infty}\left\|F_{1 i}-F^{*}\right\|_{S}=0$. Finally, this last limit implies that $\operatorname{Lim}_{i \rightarrow \infty}\left\|G_{1 i}-F^{*}\right\|_{S}=0$, which, on invoking part (iv) of Proposition 10.2, yields $\operatorname{Lim}_{i \rightarrow \infty}\left\|F_{0 i}-F^{*}\right\|_{S}=0$. Since $F_{i}=F_{0 i}$, we conclude that $\operatorname{Lim}_{i \rightarrow \infty}\left\|F_{i}-F^{*}\right\|_{S}=0$. This completes the proof.

\section{NUMERICAL VERIFICATION}

We'll illustrate the convergence results in the equal-mass case. Let $X_{n}^{*}$ denote the standardized $n$-point equal-mass collinear central configuration and $F_{n}$ its mass distribution function. From Theorem 11.4, it follows that $F_{n}$ converges uniformly to $F^{*}$ as $n$ gets large. The convergence is shown in Figures 12.1-12.5. In order, for $n=4,5,6,7$, and 8, these figures contain the graph of the step function $F_{n}$ together with that of $F^{*}$. Note also, per Theorem 11.2 , that $\Pi\left(X_{n}^{*}\right)$ is close to $\Pi^{*} \approx .26833$ and getting closer as $n$ increases. The $x$ coordinates of $X_{n}^{*}$ appear in Table 12.1 on page 2519 and have been adapted from computations due to Moeckel [6].

The initial confirmation that $X^{*}$ was the correct limiting continuous configuration came from seeing that it could accurately predict the positions of the masses in $X_{n}^{*}$. This prediction was accomplished using the approximation $X_{n}^{*} \approx C_{D}^{S}\left(X^{*} ; n\right)$ and was applied to Moeckel's data. The coordinates of $C_{D}^{S}\left(X^{*} ; n\right)$ appear in Table 12.2 on page 2520 .

This prediction process can improved and extended to the unequal mass case. We are investigating this in conjunction with other limiting mass distributions that can arise when the magnitude of one or several of the masses remains fixed as the number of masses gets large.

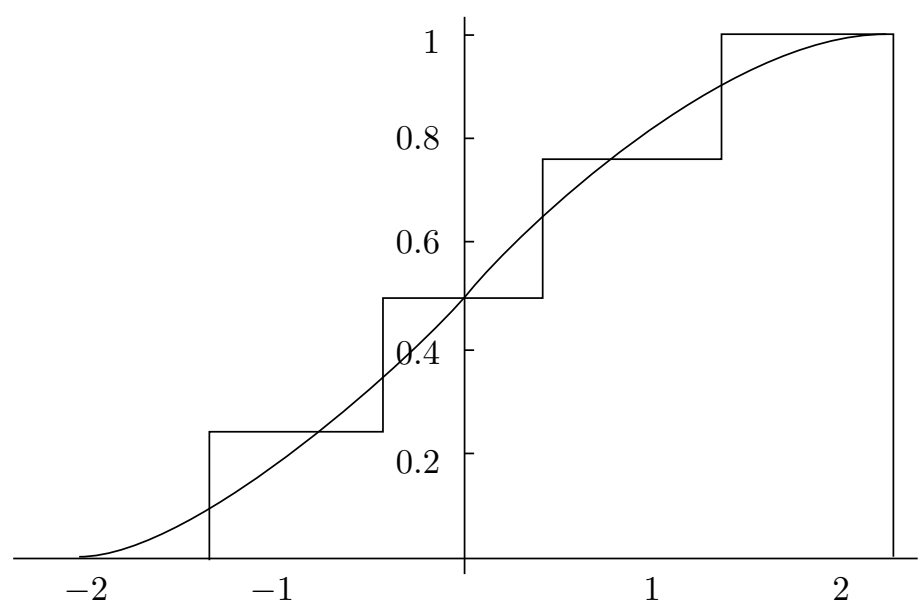

Figure 12.1. $n=4,\left\|F_{4}-F^{*}\right\|_{S} \approx .147, \Pi\left(X_{4}^{*}\right) \approx .2182, \Pi^{*}-$ $\Pi\left(X_{4}^{*}\right) \approx .0501$ 


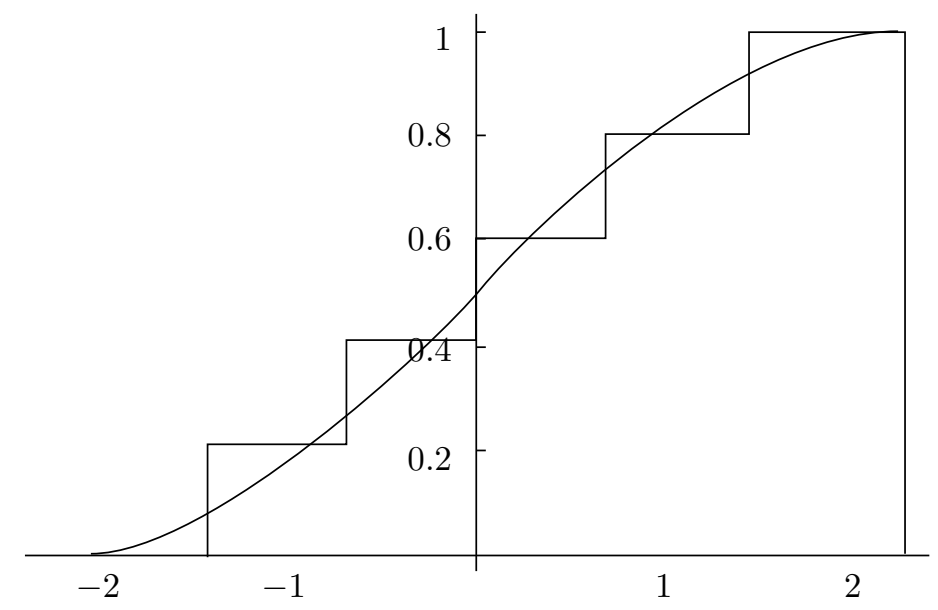

Figure 12.2. $n=5,\left\|F_{5}-F^{*}\right\|_{S} \approx .119, \Pi\left(X_{5}^{*}\right) \approx .2250, \Pi^{*}-$ $\Pi\left(X_{5}^{*}\right) \approx .0433$

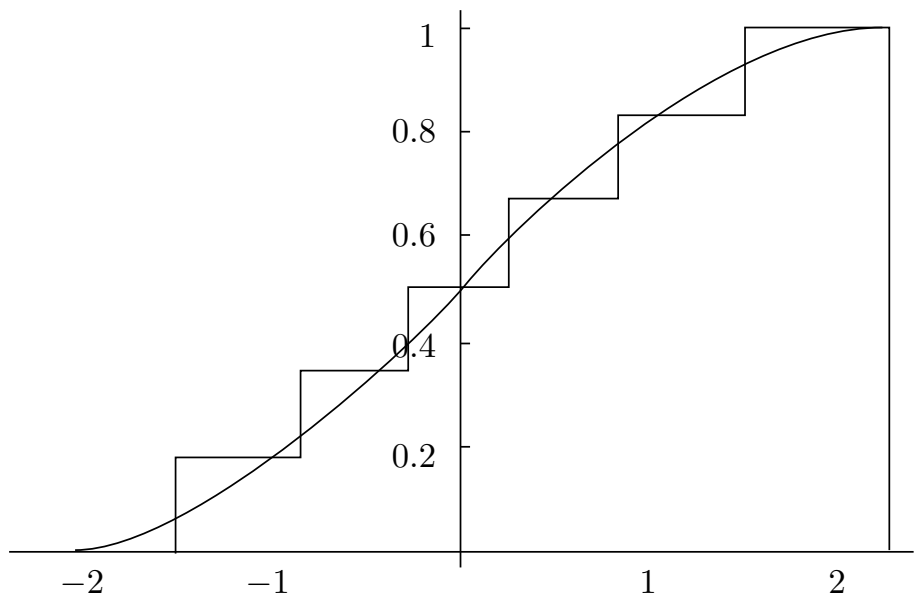

Figure 12.3. $n=6,\left\|F_{6}-F^{*}\right\|_{S} \approx .102, \Pi\left(X_{6}^{*}\right) \approx .2294, \Pi^{*}-$ $\Pi\left(X_{6}^{*}\right) \approx .0389$

TABle 12.1. Coordinates of $X_{n}^{*}$

\begin{tabular}{cccccc}
$n \mid$ & 4 & \multicolumn{1}{c}{5} & 6 & 7 & 8 \\
\hline-1.34839 & -1.43004 & -1.48930 & -1.53492 & -1.57149 \\
-0.42642 & -0.67453 & -0.84085 & -0.96193 & -1.05496 \\
0.42642 & 0.0 & -0.27378 & -0.46768 & -0.61379 \\
1.34839 & 0.67453 & 0.27378 & 0.0 & -0.20186 \\
& 1.43004 & 0.84085 & 0.46768 & 0.20186 \\
& & 1.48930 & 0.96193 & 0.61379 \\
& & & 1.53492 & 1.05496 \\
& & & & & 1.57149
\end{tabular}




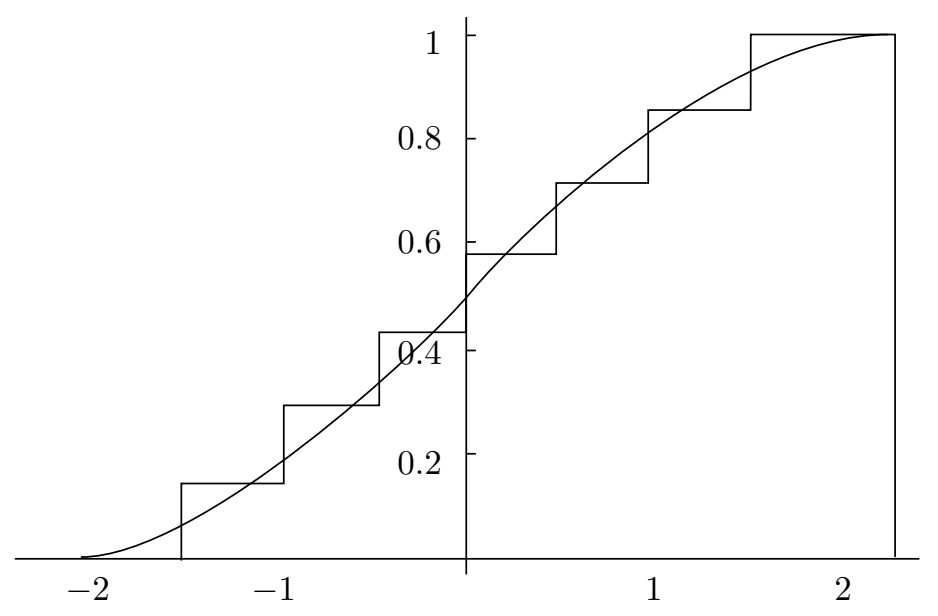

Figure 12.4. $n=7,\left\|F_{7}-F^{*}\right\|_{S} \approx .088, \Pi\left(X_{7}^{*}\right) \approx .2326, \Pi^{*}-$ $\Pi\left(X_{7}^{*}\right) \approx .0357$

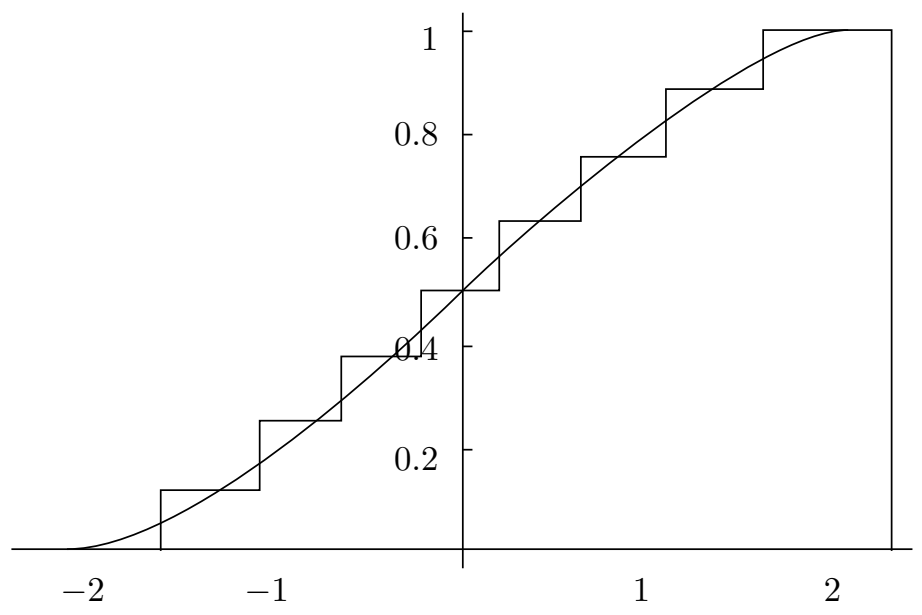

FiguRE 12.5. $n=8,\left\|F_{8}-F^{*}\right\|_{S} \approx .078, \Pi\left(X_{8}^{*}\right) \approx .2351, \Pi^{*}-$ $\Pi\left(X_{8}^{*}\right) \approx .0332$

TABLE 12.2. Coordinates of $C_{D}^{S}\left(X^{*} ; n\right)$

\begin{tabular}{cccccc}
$n \mid$ & \multicolumn{1}{c}{5} & 6 & 7 & 8 \\
\hline-1.35397 & -1.44214 & -1.50765 & -1.55895 & -1.60062 \\
-0.40836 & -0.64826 & -0.81178 & -0.93279 & -1.02718 \\
0.40836 & 0.0 & -0.26080 & -0.44672 & -0.58819 \\
1.35397 & 0.64826 & 0.26080 & 0.0 & -0.19224 \\
& 1.44214 & 0.81178 & 0.44672 & 0.19224 \\
& & 1.50765 & 0.93279 & 0.58819 \\
& & & 1.55895 & 1.02718 \\
& & & & 1.60062
\end{tabular}




\section{Appendix A}

Lemma A1. Let $f(x)$ be non-negative on $(-\infty, \infty)$ and have a set of discontinuities which has measure 0 . Then, if

$$
\int_{-\infty}^{\infty} f^{2}(x) d x<\infty, \operatorname{Lim}_{t \rightarrow 0} \int_{-\infty}^{\infty} f(x+t) f(x) d x=\|f\|_{2}^{2} .
$$

Proof. Since $\int_{-\infty}^{\infty} f^{2}(x+t) d x=\int_{-\infty}^{\infty} f^{2}(x) d x$. The Schwarz inequality yields

$$
\int_{-\infty}^{\infty} f(x+t) f(x) d x \leq \sqrt{\int_{-\infty}^{\infty} f^{2}(x+t) d x} \sqrt{\int_{-\infty}^{\infty} f^{2}(x) d x}=\int_{-\infty}^{\infty} f^{2}(x) d x,
$$

and thus $\int_{-\infty}^{\infty} f(x+t) f(x) d x$ achieves its maximum at $t=0$.

Now let $t_{n} \rightarrow 0$. From Fatou's lemma, $\int_{-\infty}^{\infty} \operatorname{LimInf}_{n \rightarrow \infty} f\left(x+t_{n}\right) f(x) d x \leq$ $\operatorname{LimInf}_{n \rightarrow \infty} \int_{-\infty}^{\infty} f\left(x+t_{n}\right) f(x) d x$. But $\operatorname{LimInf}_{n \rightarrow \infty} f\left(x+t_{n}\right) f(x)=f^{2}(x)$ a.e. Thus, $\int_{-\infty}^{\infty} f^{2}(x) d x \leq \operatorname{LimInf}_{n \rightarrow \infty} \int_{-\infty}^{\infty} f\left(x+t_{n}\right) f(x) d x \leq \int_{-\infty}^{\infty} f^{2}(x) d x$. Further, $\operatorname{LimSup}_{n \rightarrow \infty} \int_{-\infty}^{\infty} f\left(x+t_{n}\right) f(x) d x \leq \int_{-\infty}^{\infty} f^{2}(x) d x$. As a result,

$$
\operatorname{Lim}_{n \rightarrow \infty} \int_{-\infty}^{\infty} f\left(x+t_{n}\right) f(x) d x=\int_{-\infty}^{\infty} f^{2}(x) d x,
$$

thus establishing the lemma.

Lemma A2. Let $f(x)$ be non-negative on $(-\infty, \infty)$ and have a set of discontinuities which has measure 0 . Then, if $\int_{-\infty}^{\infty} f^{2}(x) d x<\infty$ and $h(t)$ is defined by $h(t)=\int_{-\infty}^{\infty} f^{2}(x) d x-\int_{-\infty}^{\infty} f(x+t) f(x) d x$

$$
\operatorname{Lim}_{\varepsilon \rightarrow 0^{+}} \frac{\int_{\varepsilon}^{1} t^{-1} h(t) d t}{-\log (\varepsilon)}=0 .
$$

Proof. For small $\varepsilon>0$ choose $\delta=\frac{1}{\log (1 / \varepsilon)}$, and set $\tau=\operatorname{Sup}_{0 \leq t \leq \delta} h(t)$ and $m=\operatorname{Sup}_{0 \leq t \leq 1} h(t)$. We have $\operatorname{Lim}_{\varepsilon \rightarrow 0} \frac{\varepsilon}{\delta}=0, \operatorname{Lim}_{\varepsilon \rightarrow 0} \delta=0$, and $\operatorname{Lim}_{\varepsilon \rightarrow 0} \tau=0$ by Lemma A1. Writing $\int_{\varepsilon}^{1} \frac{h(t)}{t} d t=\int_{\varepsilon}^{\delta} \frac{h(t)}{t} d t+\int_{\delta}^{1} \frac{h(t)}{t} d t$ for small $\varepsilon$ yields $0 \leq$ $\int_{\varepsilon}^{1} \frac{h(t)}{t} d t \leq \tau(\log (\delta)-\log (\varepsilon))+m(-\log (\delta)) \leq \tau(-\log (\varepsilon))+m(-\log (\delta))$, and $0 \leq \frac{\int_{\varepsilon}^{1} \frac{h(t)}{t} d t}{-\log (\varepsilon)} \leq \tau+m \frac{\log (\log (1 / \varepsilon))}{\log (1 / \varepsilon)}$. This completes the proof, since the right side of the last inequality tends to 0 as $\varepsilon \rightarrow 0$.

\section{Proof of Proposition 2.1}

Proof. To show $\operatorname{Lim}_{n \rightarrow \infty} \frac{\int_{-\infty}^{\infty} \int_{y+\frac{c}{n}}^{\infty} \frac{f(x) f(y) d x d y}{x-y}}{\log (n)}=\int_{-\infty}^{\infty} f^{2}(x) d x$, we begin by letting $\varepsilon=\frac{c}{n}>0$ and setting $P(f ; \varepsilon)=\int_{-\infty}^{\infty} \int_{y+\varepsilon}^{\infty} \frac{f(x) f(y) d x d y}{x-y}$. Using $\int_{y+\varepsilon}^{\infty} \frac{f(x) d x}{x-y}=$ $\int_{\varepsilon}^{1} \frac{f(y+t) d t}{t}+\int_{1}^{\infty} \frac{f(y+t) d t}{t}$ gives

$$
P(f ; \varepsilon)=\int_{-\infty}^{\infty}\left(\int_{1}^{\infty} \frac{f(y+t) d t}{t}\right) f(y) d y+\int_{-\infty}^{\infty}\left(\int_{\varepsilon}^{1} \frac{f(y+t) d t}{t}\right) f(y) d y .
$$

Letting $V$ denote the value of the first double integral in (1), and interchanging the order of integration in the second give $P(f ; \varepsilon)=V+\int_{\varepsilon}^{1} \frac{1}{t}\left(\int_{-\infty}^{\infty} f(y) f(y+t) d y\right) d t$. As a result, we can write $P(f ; \varepsilon)=V+\int_{\varepsilon}^{1} \frac{1}{t}\left(\int_{-\infty}^{\infty}\left(f(y) f(y+t)-f^{2}(y)\right) d y\right) d t+$ 
$\int_{\varepsilon}^{1} \frac{1}{t}\left(\int_{-\infty}^{\infty} f^{2}(y) d y\right) d t$. Using the notation of Lemma A2 yields $P(f ; \varepsilon)=V-$ $\int_{\varepsilon}^{1} \frac{h(t)}{t} d t-\log (\varepsilon)\|f\|_{2}^{2}$. Equivalently,

$$
\begin{aligned}
& \frac{1}{\log (n)} \int_{-\infty}^{\infty} \int_{y+c / n}^{\infty} \frac{f(x) f(y) d x d y}{x-y} \\
& \quad=\frac{V}{\log (n)}-\left(\frac{-\log (\varepsilon)}{\log (n)}\right)\left(\frac{\int_{\varepsilon}^{1} t^{-1} h(t) d t}{-\log (\varepsilon)}\right)+\left(\frac{-\log (\varepsilon)}{\log (n)}\right)\|f\|_{2}^{2} .
\end{aligned}
$$

By letting $n$ go to infinity, applying Lemma A2, and using the fact that

$$
\operatorname{Lim}_{n \rightarrow \infty}\left(\frac{-\log (\varepsilon)}{\log (n)}\right)=1
$$

we see that the above equality yields the theorem.

\section{Appendix B}

Let $n$ be greater than 1 , and $X=X_{A}$ be an $n$-point configuration with no restriction on the total mass of $X$, and let $S_{1}$ be a non-empty set of consecutive integers drawn from the set $\{1,2, \ldots, n\}$, and $S_{2}$ be the remaining integers in $\{1,2, \ldots, n\}$.

Let $X_{1}$ and $X_{2}$ be subconfigurations of $X$ defined as follows: $X_{1}$ is the configuration consisting of those point masses of $X$ whose subscripts are in $S_{1}$, and $X_{2}$ those point masses of $X$ with subscripts in $S_{2}$. $M_{1}$ will denote the total mass of $X_{1}$ and $\bar{x}_{1}=\sum_{i \in S_{1}} \frac{a_{i}}{M_{1}} x_{i}$, the center of mass of $X_{1} . \widetilde{X}_{1}=\left(\bar{x}_{1} ; M_{1}\right)$ is the single point configuration obtained by coalescing the point masses of $X_{1}$ into a single point with mass $M_{1}$ at $\bar{x}_{1}$. Finally, let $\widetilde{X}$ be the configuration obtained from $X$ by replacing the point masses in $X_{1}$ by $\widetilde{X}_{1}$ and leaving the other point masses of $X$ unchanged.

Proposition B1 implies that if we partition a discrete configuration into disjoint subsets of successive point masses and combine each subset into a single point mass at the center of mass of the subset, then we produce a new configuration with potential no greater than that of the original configuration. Further, if any subset contains more than a single point, the new configuration has fewer points and lower potential than that of the original.

Proposition B1. $U(\widetilde{X}) \leq U(X)$, with strict inequality if $X_{1}$ contains more than a single point.

Proof. Since the potential of a configuration consisting of a single point is 0 , if $X_{2}$ is empty, we have $U(\widetilde{X})=0<U(X)$. Otherwise, using the formulations $U(X)=U\left(X_{1}\right)+U\left(X_{2}\right)+C\left(X_{1}, X_{2}\right)$ and $U(\widetilde{X})=U\left(\widetilde{X}_{1}\right)+U\left(X_{2}\right)+C\left(\widetilde{X}_{1}, X_{2}\right)$, where $C$ denotes the cross potential (see Definition 9.2), we find $U(X)-U(\widetilde{X})=$ $U\left(X_{1}\right)+C\left(X_{1}, X_{2}\right)-C\left(\widetilde{X}_{1}, X_{2}\right)$. To complete the proof we need only show that $C\left(\widetilde{X}_{1}, X_{2}\right) \leq C\left(X_{1}, X_{2}\right)$.

Let $l$ be the smallest integer in $S_{1}$ and $k$ the largest, and let $S_{22}$ be those integers in $S_{2}$ which exceed $k$, and $S_{21}$ those in $S_{2}$ which are less than $l$. Define $g(x)$ according to $g(x)=\sum_{j \in S_{22}} \frac{M_{1} a_{j}}{x_{j}-x}+\sum_{j \in S_{21}} \frac{M_{1} a_{j}}{x-x_{j}}$, where one of the sums may be non-existent if the index set is empty. Since $j \in S_{21}$ implies $x_{j}<x_{l}$ and $j \in S_{22}$ implies $x_{j}>x_{k}$, it follows that $g$ is continuous with continuous first and second derivatives on some open interval $I$ which contains the interval $\left[x_{l}, x_{k}\right]$. Further, $g^{\prime \prime}(x)>0$ on $I$, and hence, $g$ is convex on $I$. 
Since $C\left(\widetilde{X}_{1}, X_{2}\right)=g\left(\bar{x}_{1}\right)$, and

$$
C\left(X_{1}, X_{2}\right)=\sum_{i \in S_{1}} \frac{a_{i}}{M_{1}}\left[\sum_{j \in S_{21}} \frac{a_{j} M_{1}}{x_{i}-x_{j}}+\sum_{j \in S_{22}} \frac{a_{j} M_{1}}{x_{j}-x_{i}}\right]=\sum_{i \in S_{1}} \frac{a_{i}}{M_{1}} g\left(x_{i}\right),
$$

the convexity of $g$ on $I$ yields $C\left(\widetilde{X}_{1}, X_{2}\right) \leq C\left(X_{1}, X_{2}\right)$.

\section{REFERENCES}

1. G. Buck, 1991, The collinear central configuration of $n$ equal masses, Celestial Mech. Dynam. Astronom. 51, 305-317. MR 92k:70013

2. L. Euler, 1767, De moto recilineo trium corporum se mutuo attahentium, Novi Comm. Acad. Sci. Imp. Petrop. 11, 144-151.

3. P. W. Lindstrom, 1996, Limiting mass distributions of minimal potential central configurations, Hamiltonian Dynamics and Celestial Mechanics, Contemporary Mathematics, vol. 198, Amer. Math. Soc., Providence, RI, pp. 109-129. MR 97g:70015

4. K. R. Meyer and G. R. Hall, 1992, Introduction to Hamiltonian Dynamical Systems and the $N$-Body Problem, Springer-Verlag. MR 93b:70002

5. R. Moeckel, 1990, On central configurations, Math. Z. 205, 499-517. MR 92b:70012

6. R. Moeckel, Some relative equilibria of $n$ equal masses, $n=4,5,6,7,8$, unpublished.

7. F. R. Moulton, 1910, The straight line solutions of the problem of $N$ bodies, Ann. Math., II. Ser. $12,1-17$.

8. D. G. Saari, 1980, On the role and properties of $n$ body central configurations, Celestial Mech. 21, 9-20. MR 81a:70016

Department of Mathematics, Saint Anselm College, Manchester, New Hampshire 03102 